\title{
Deep-Plane Approach to the Vertical Platysma Advancement: Technical Modifications and Nuances over 25 Years
}

\author{
Neil A. Gordon, MD ${ }^{1,2}$ Tareq G. Sawan, MBChB ${ }^{1}$ \\ ${ }^{1}$ Division of Otolaryngology - Head and Neck Surgery, Department of \\ Surgery, Yale University School of Medicine, New Haven, \\ Connecticut \\ 2 New England Surgical Center, The Retreat at Split Rock, Wilton, \\ Connecticut
}

Facial Plast Surg 2020;36:358-375.

\begin{abstract}
Keywords

- deep plane

- facelift

- rhytidectomy

- midface

- facial aging

- aging neck

- vertical platysma advancement

- aesthetic surgery
\end{abstract}

Address for correspondence Tareq G. Sawan, MBChB, Division of Otolaryngology - Head and Neck Surgery, Department of Surgery, Yale School of Medicine, Yale Physicians Building, 4th Floor 800 Howard Avenue, New Haven, CT 06510 (e-mail: tareq.sawan@yale.edu).
The lead author (N. G.) has been an early proponent of the deep-plane rhytidectomy and the concepts that support this technique, including the "surgical model" of soft tissue redundancy over the "volume model" of volume loss, as the main component of facial aging. ${ }^{1}$ Based on a model developed by the lead author (N. G.), and recent studies further supporting this premise, there is no significant volume loss in superficial and deep fat compartments with aging but is actually gained in dependent areas, consistent with soft tissue redundancy. ${ }^{2,3}$ These findings, in addition to intraoperative evidence, demonstrate the predominant role of soft tissue redundancy in the aging process. It is the author's view that the perpetuation of the volume loss theory has been multifactorial, mainly due to the illusion of volume loss created by the appearance of hollows and folds from the redundant superficial soft tissue envelope ${ }^{1}$ and partly due to the neglected contribution of facial skeletal aging, especially in the older patient cohort. This has been further validated by the inadequacy and limitations of surgical techniques that were not anatomically based. This resulted in many of the suboptimal aesthetic issues that caused the questioning of the "surgical model."

The anatomical and embryologic validity of the deep-plane rhytidectomy is revealed in the history of the procedure. Since the early 1900s, the initial technique of subcutaneous undermining and resection of the skin under tension provided aesthetically obvious and short-lived results given the unnatural skin tension, inherit elasticity of skin, and failure to address the deeper components of the face. Skoog, ${ }^{4}$ in the early 1970s, applied his anatomical observations of a superficial fascial layer
Issue Theme Controversies in Facelifting; Guest Editor: Fred G. Fedok, MD, FACS
Copyright (c) 2020 by Thieme Medical Publishers, Inc., 333 Seventh Avenue, New York, NY 10001, USA. Tel: +1(212) 760-0888. ISSN 0736-6825. 
into a subfascial dissection, which maintained the attachment of the overlying skin and subcutaneous tissue to the flap. This fascial layer was further described in 1974 by Mitz and Peyronie $^{5}$ using cadaveric dissections; they termed it the superficial muscular aponeurotic layer (SMAS). The SMAS was found to be continuous with the frontalis superiorly, temporoparietal fascia superolaterally, and platysma inferiorly. Although they noted that the skin and subcutaneous fat were adherent to this layer through "septations," the significance of this finding was overlooked. Skoog's original description of the sub-SMAS technique addressed the jawline but with very limited impact on the midface and neck. Hamra, ${ }^{6,7}$ in the early 1990s, advanced this technique by continuing the sub-SMAS dissection superiorly to incorporate the medial cheek fat compartment, maintaining its attachment to the skin, in a musculocutaneous flap-the deep-plane rhytidectomy.

The original technique described a dissection from the angle of the mandible to the orbicularis oculi. Hamra correctly described the advantages of the deep-plane dissection in the flap's ability to withstand substantial traction without creating unnatural surface tension or distortion commonly seen in SMAS procedures. He also noted that this approach enabled direct access to and lysis of the zygomatic cutaneous ligament, a major facial retaining ligament. This allowed better mobilization of the superficial soft tissue envelope, containing the majority of facial fat, without the necessity of surface tension. Rohrich and Pessa's ${ }^{8}$ detailed cadaveric anatomical descriptions of the superficial soft tissue envelope further validated the anatomical basis supporting the deep-plane technique. Their dissection demonstrated that the superficial soft tissue envelope, the SMAS to skin, consisted of fat compartments divided by fibrous septations. These septae provide both vascular supply and structural support. Importantly, these septal connections explain why keeping the superficial soft tissue envelope intact creates a mechanical advantage that allows traction on the underbelly (SMAS/platysma) layer alone to mobilize the entire anatomical unit without creating surface tension. All SMAS procedures separate this anatomical relationship, thus eliminating this mechanical advantage. Since the goal in the surgical model of facial rejuvenation is to maximally reduce soft tissue redundancy without creating surface tension, further evidence in support was provided in Litner and Adamson's study. $^{9}$ They compared SMAS techniques with deep-plane rhytidectomy in the same patient and the on same side by measuring redundancy created and objectively defined the deep-plane rhytidectomy's superiority.

Additional technical advances not originally described have subsequently been defined by the lead author and others, allowing us to approach other components of aging as well as increase the degree and longevity of the aesthetic outcome. These advances are either related to the access provided by the deep-plane dissection to treat other components of aging or by increasing the sub-SMAS/platysma flap dissection and releasing additional facial ligaments, increasing the degree and extent of soft tissue repositioning and excision.

We recount the multiple advantages of this approach. First, the deep plane allows direct assessment and treat- ment of deeper aging issues such as pseudoherniation of buccal fat for the treatment of jowling. Second, the procedure optimizes midfacial mobilization; since the SMAS is contiguous with the platysma muscle in the neck, subSMAS mobilization of the midface and superolateral resuspension of the SMAS/platysma unit provide significant and long-lasting neck rejuvenation. Third, extended subplatysma dissection below the mandible and selective release of the angle of the mandible and cervical retaining ligaments can be included for aggressive neck treatment. Fourth, because of variations in flap design and access, facial contour can be controlled through volumization to increase malar width and/or taper the lower midface width by soft tissue as well as parotid hypertrophy contouring. Fifth, since the deep-plane flap resuspension confines tension to the platysma/SMAS fascia, this technique is preferable for revision rhytidectomy procedures in which avoiding or reversing skin tension is paramount to the procedural success (-Fig. 1). Sixth, since the dissection is performed in a relatively avascular plane, and the soft tissue mobilized is well vascularized, bruising is minimized and healing parameters are optimized (-Fig. 2). This allows marginal candidates, such as smokers and diabetics, to be considered under certain circumstances. ${ }^{10}$ Finally, the tension-free skin closure minimizes complications and ensures longer-lasting natural rejuvenation.

In this article, we will describe the technical modifications, advances, and variations of Hamra's original description that we have named the "vertical plastysma advancement."

\section{Anatomy}

Our preference for the deep-plane approach as a safe and effective technique stems from understanding the relevant embryology and anatomy of the face. Viewing the layers of the face (-Fig. 3), we see the superficial muscular aponeurotic system (SMAS) forming the deep portion of the superficial soft tissue envelope, with the majority of facial fat distributed homogeneously superficial to this layer. ${ }^{8}$ This embryologic boundary is contiguous from the platysma in the neck to the galea in the forehead. Below this layer is the superficial layer of the deep cervical fascia, covering all the deeper embryologic structures (-Fig. 4) such as the facial nerve, masseter muscle, and buccal fat pad. The potential space located between these layers represents the embryologic cleavage plane of the midface that defines the deep plane. Dissection in this potential space in the midface is relatively avascular, and the facial nerve is securely protected by the deep cervical fascia. Additionally, the SMAS fascia fuses with the superficial layer of the deep cervical fascia at the parotid gland, fixating the facial soft tissue laterally. It is poorly defined superiorly over the facial mimetic muscles such as the zygomatic and orbicularis muscles. Thus, the majority of the malar fat pad sits on this superior group of facial mimetic muscles.

Crossing through these planes are two main facial ligaments-at the body of the zygoma are the zygomatic cutaneous ligaments (McGregor's patch) and inferiorly on the 

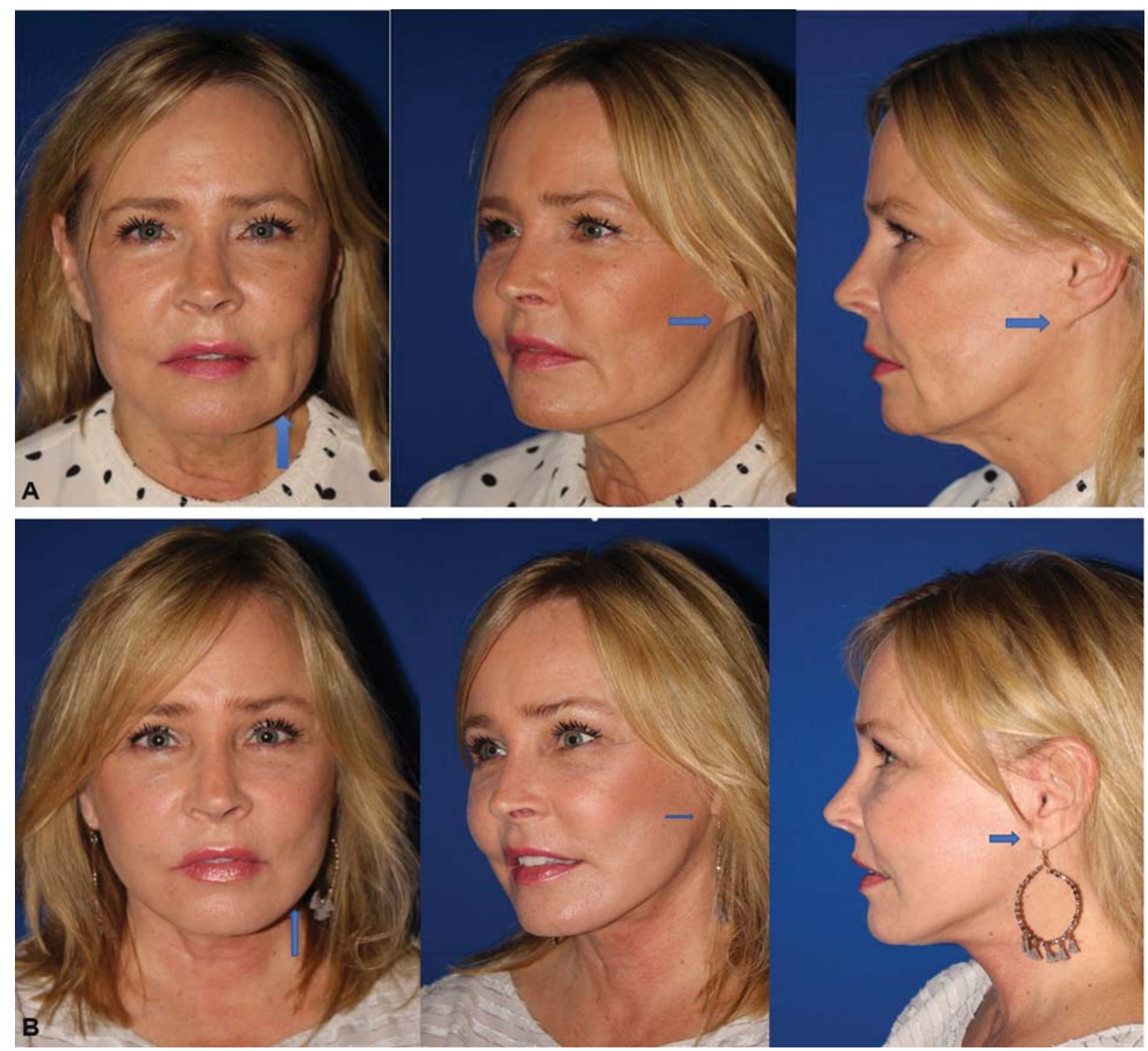

Fig. 1 (A) Preoperative and (B) postoperative photographs of a 62-year-old female after multiple superficial muscular aponeurotic layer (SMAS) rhytidectomy, buccal fat removal, fat injections, and facial fillers. She was treated with revision rhytidectomy, endo/coronal forehead lift, lower blepharoplasty, and periocular and perioral erbium:yttrium aluminum garnet laser skin resurfacing. The deep-plane technique is optimal in revision cases since skin tension can be released and transferred to the underlying fascia. This allows both a natural outcome while repairing prior soft tissue deficits and pixie ear deformity. Despite multiple SMAS rhytidectomy, the surgical model was not wrong but executed suboptimally.

medial aspect of the mandible are the mandibular ligaments, which are the sole anchors of the facial soft tissue envelope (-Fig. 4). Musculocutaneous ligaments also course from the medial edge of the masseter muscle to the dermis in the midface. The cervical retaining ligaments extend from the angle of the mandible inferiorly along the posterior platysma anchoring the muscle to the sternocleidomastoid muscle. The deep plane is very well defined inferiorly along the masseter muscle due to the presence of platysma muscle in the SMAS fascia and the presence of fibroareolar tissue consistent with a plane or potential space. The retaining ligaments along the angle of the mandible continue inferiorly as cervical retaining ligaments: the marginal mandibular and cervical branches of the facial nerve are in this field but protected by the deep cervical fascia. Superiorly, the deep plane is more poorly defined as the fascia thins out at the level of the facial mimetic muscles.

\section{Evaluation}

Evaluation of a surgical candidate should include the standard medical clearance for a patient undergoing general anesthesia. We prefer that the patient be intubated under a propofol-based anesthetic. Facelift procedures are often combined with browlifts and eyelid procedures. Physical evaluation should be based on the specific combination of procedures to be performed. The presence and degree of aging changes, such as the nasolabial folds, jowling, platysma banding, ptotic submandibular triangles, submental fat deposition, and festoons, should be established prior to the deep-plane facelift. ${ }^{11,12}$ Normal anatomical variances, including facial dimples and the hyoid/mental relationship, are noted during the initial evaluation.

From the perspective of the facial plastic surgeon, the importance of defining face type by palpation of the facial 

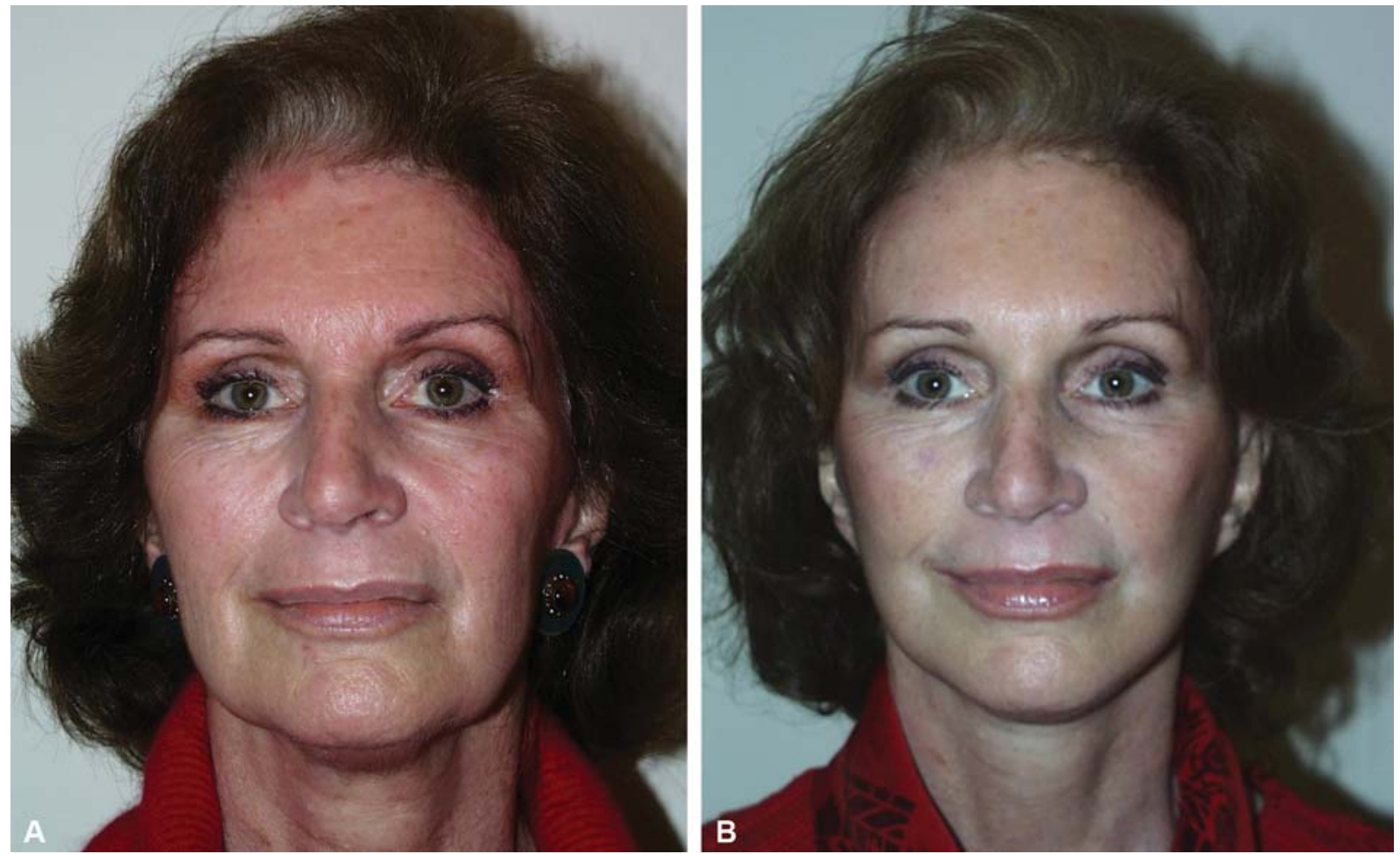

Fig. 2 A 67-year-old woman (A) before and (B) after 14 days of revision deep-plane rhytidectomy and browlift.
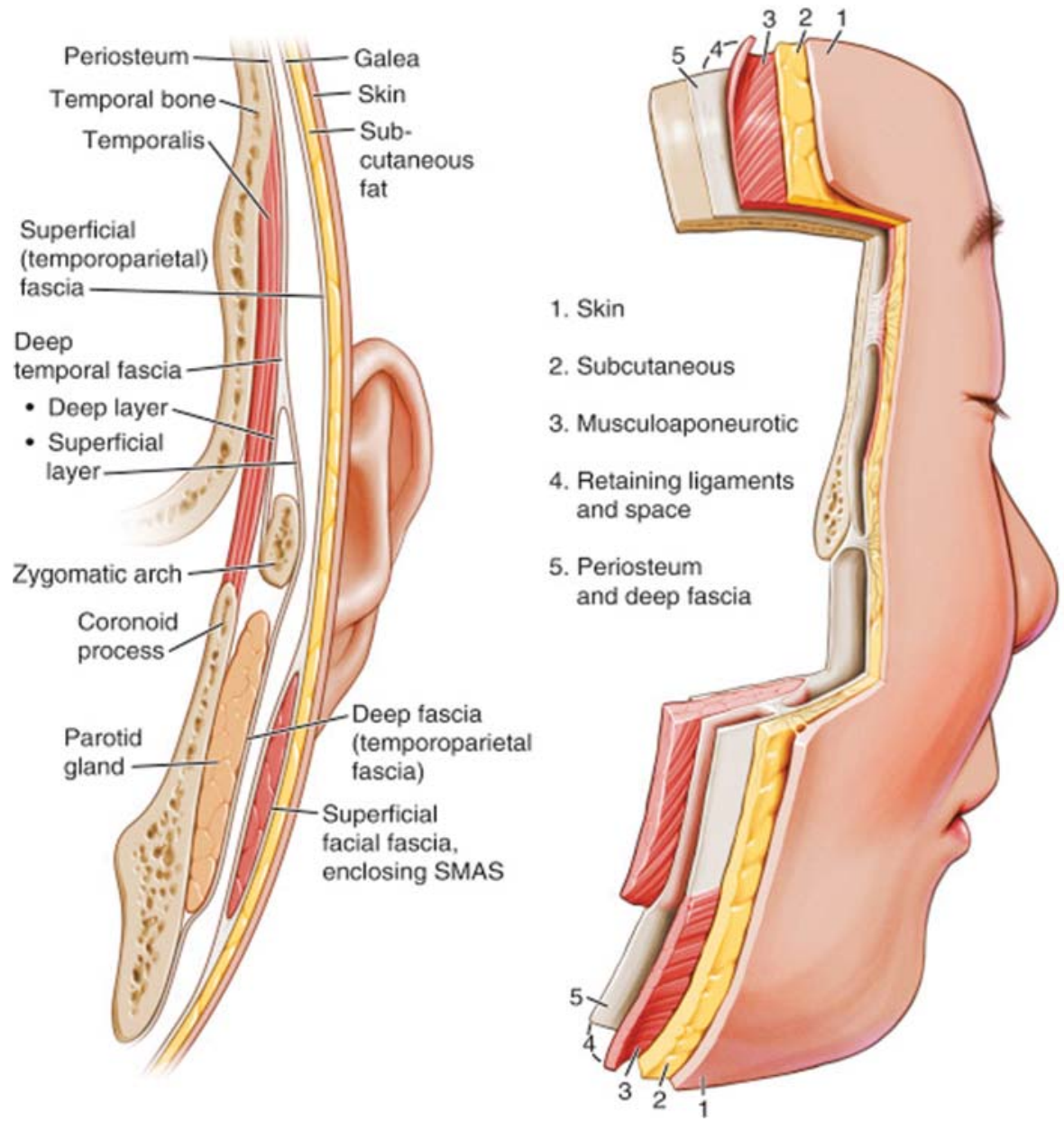

Fig. 3 Layers of the face and their relationship with the musculoskeletal framework. (Reproduced with permission of Niamtu J III. Facelift surgery (cervicofacial rhytidectomy). In: Cosmetic Facial Surgery. Philadelphia, PA: Elsevier; 2018.) 


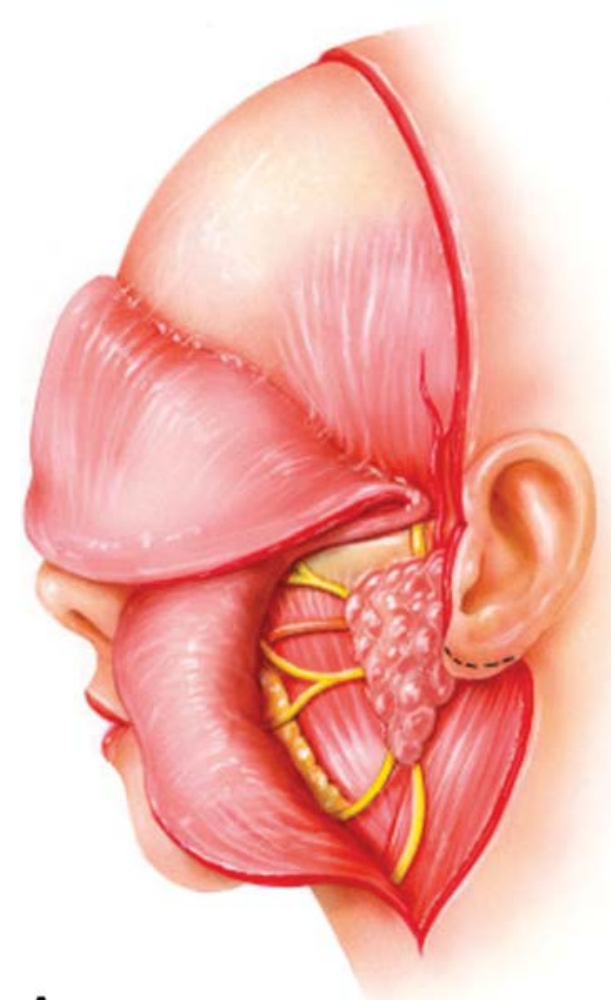

A

Fig. 4 (A) The deep plane of the face is the embryologic cleavage plane separating the superficial soft tissue envelope from the deeper structural aspects of the face that are bounded by the deep cervical fascia. (B) Retaining ligaments of the face with the superficial muscular aponeurotic layer elevated. Both the zygomatic ligament (McGregor's patch) superiorly and the mandibular ligament inferiorly are strong ligaments with periosteal attachments. (Reproduced with permission of Sykes JM, Suárez GA, Trevidic P, Cotofana S. Applied facial anatomy. In: Master Techniques in Facial Rejuvenation. Edinburgh: Elsevier; 2018.)

soft tissue should be emphasized. Compliant faces will often manifest the effects of gravity at an earlier stage and create more soft tissue mobilization and redundancy, thus requiring careful hairline planning. Stiff, noncompliant faces can be more difficult to dissect, but these will typically maintain their outcomes for a longer period of time. Thicker soft tissue will require more lateral/periauricular soft tissue contouring after the flap is mobilized and fixated. In thin faces, the deep plane will be more proximal to the subcutaneous tissue plane. Presence of jowling disproportionate to the degree of neck changes is often indicative of buccal fat pseudoherniation. Deeper marionette lines at the jawline can indicate the need for mandibular ligament release.

When evaluating the neck, differentiating between superficial soft tissue (from platysma to skin) and subplatysmal agerelated changes is vital to producing optimal, predictable neck outcomes. This significance is revealed when optimal surgical treatment of the platysma unit is achieved in a patient with untreated subplatysmal age-related changes. The better the result in the superficial soft tissue layer, the more accentuated the subplatysmal age-related changes may become after rhytidectomy. On the frontal view of the neck, the platysma component is typically medial, often continuing below the hyoid bone in the aging neck. The ptotic or thickened anterior digastric muscles, and/or ptotic submandibular gland, are more laterally located and are confined superior to the lateral aspect of the hyoid bone. Subplatysmal fat can exist both medially and extend laterally, adding to the appearance of the aged neck. Asking the patient to flex the platysma muscle can be helpful in defining which neck components are most prominent preoperatively. This examination may be very difficult in the severely aged or fatty neck as anatomical landmarks can become obscured (-Fig. 5).

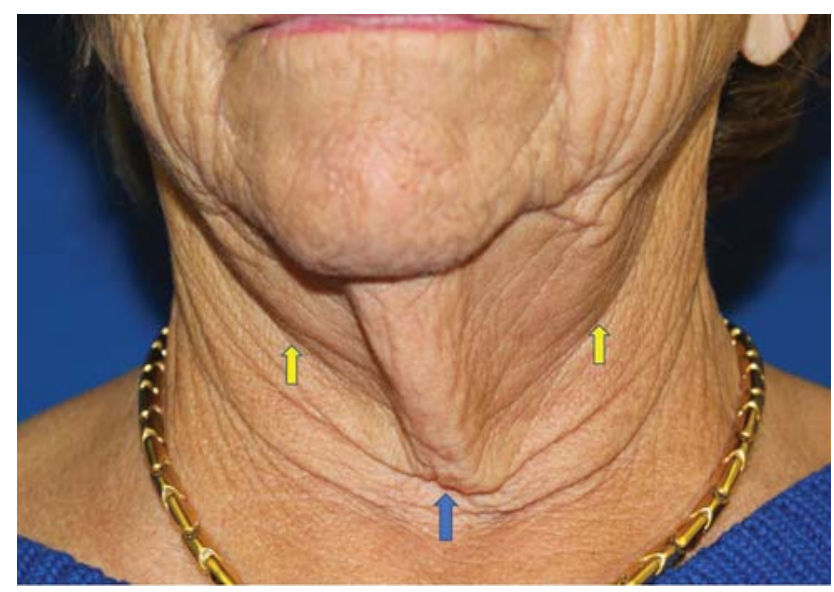

Fig. 5 The submental view can be helpful is differentiating between superficial/ medial neck (blue arrow) and deep/paramedian neck structures (yellow arrows). The superficial neck is treated with superficial soft tissue envelope, midface mobilization, and direct platysmal resection/embrocation, whereas the deep neck requires subplatysmal treatment and lateral cervical ligament release and resuspension. 
Quality of neck skin is also an important predictor of neck outcome and nuances of the anticipated preplatysmal neck dissection portion of the procedure. In most cases, the majority of aging is confined to the superficial soft tissue envelope. In patients aged 65 years and above, a greater, but still secondary, proportion of aging changes can be both skeletal and deep tissue related.

Complete neurologic evaluation should document both trigeminal and facial nerve status. Static and dynamic images should be obtained in frontal, three quarter, and lateral views. Ear position and shape, facial asymmetries, and hairlines should be documented. Dynamic images should document patterns of muscle action.

\section{Patient Perspective}

Patients desire a maximal aesthetic outcome with the least extensive process possible. The career and social demands of many patients require them to be cosmetically presentable in public within 2 weeks. An individual's aesthetic goals should be balanced with their stage of aging and any anatomical limitations. We find conducting a mirror examination, where the patient's face is mobilized while they look in the mirror, effective in indicating the potential outcomes as well as communicate what procedures will address what aging issues. Before and after photographs of the contemplated procedure(s) with similarly profiled previous patients are helpful in establishing realistic expectations of outcomes. Aging changes that may be untreated, regardless of technique, such as festoons or ptotic submandibular triangles, should be discussed so that appropriate patient expectations can be defined. Risks and benefits should be discussed prior to any contemplated procedure. Minimally invasive procedures and their limitations can be presented as alternatives, if appropriate.

\section{Surgical Procedure}

The importance of detailed surgical planning is crucial in aesthetic surgery. Intraoperative access to preoperative photographs is also essential. Although most of our deep-plane facelifts are combined with other procedures, we will describe only the facelift portion of the procedure below.

We prefer to perform the procedures under a propofolbased general anesthetic. Bleeding is controlled throughout the procedure by a surgical field injection of a $1: 1 \mathrm{mix}$ of $1 \%$ lidocaine with 1:100,000 epinephrine and 0.5\% bupivacaine with 1:200,000 epinephrine combined with hypotensive anesthetic techniques. We recently began adding tranexamic acid 1,000 mg to the local anesthetic and, in cases with greater bleeding risk, to the intravenous solution as well. Perioperative antibiotics covering skin flora and one dose of intravenous steroids are given prior to incision. Except for a short-acting agent for anesthesia induction, we do not use muscle relaxants.

Once the patient has been anesthetized, we proceed to cut the hair and mark accordingly (-Fig. 6). A retrotragal incision is typically used in females because the deep-plane procedure avoids tension on the incision lines, maintaining normal ear and

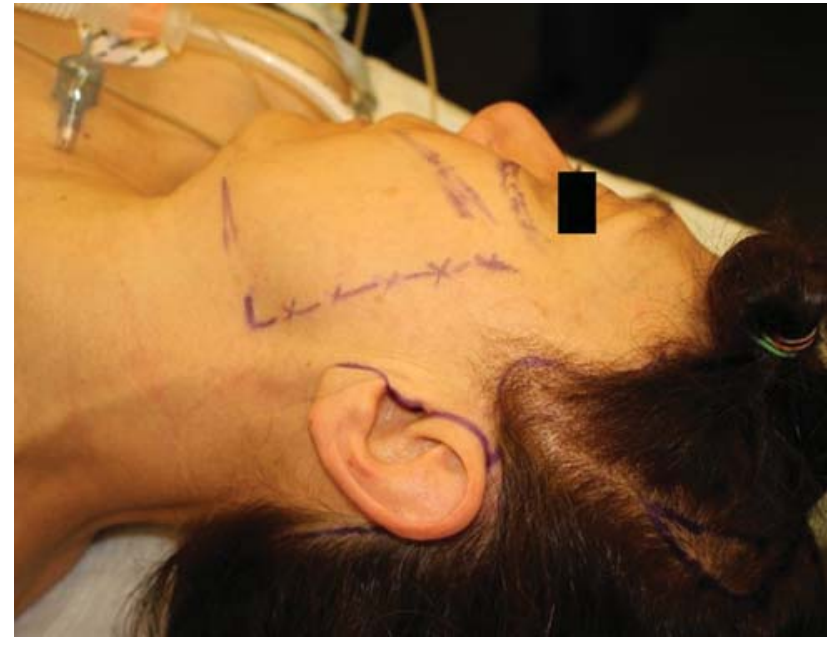

Fig. 6 Marked hairline incision for the maximal preservation of hairline. The diagonal line extending from the angle of the mandible to the lateral canthus demonstrates the surface landmark of deepplane entry. This entry point will vary based on specific case needs.

tragal architecture. Pretragal incisions are necessary in men to avoid moving hair-bearing skin onto the tragus. Note the position of the earlobe while the patient is supine as it may differ from the photographs and, once released, will not be available for reference (-Fig. 7). Since there is a significant degree of soft tissue mobilization using the deep-plane technique, even in younger patients, we prefer to use temporal hairline incisions to avoid superior and posterior displacement of the temporal hair tuft ( $\mathbf{F i g . ~ 8 )}$ ). Based on our prediction of soft tissue mobilization, we will design the postauricular limb more superiorly, with a more acute angle (compared with the antihelix), for patients with relatively compliant soft tissue. We will also mark the inferior limit of the posterior hairline at the nape of the neck to ensure incision line camouflage. For patients who have less compliant soft tissue or with revision cases, the postauricular limb is based more inferiorly with a more obtuse angle. ${ }^{13}$ When a significant degree of soft tissue is mobilized in the midface and neck, there is a greater need to extend incisions to accommodate redundant soft tissue and avoid dog ears or tissue bunching.

Incisions are made using a no. 10 blade, ensuring to bevel at exposed hairlines for the preservation of hair follicles, such as the anterior temporal tuft. We carefully incise the skin at the edge of the tragus to hide the incision while avoiding the unnatural skin drape that would occur upon closing an incision placed too far retrotragally. Cautery is not used at bleeding hairlines to preserve the hair follicles. Application of a cold lap pad or sponge will often tamponade hairline bleeding.

Sharp dissection then proceeds in the preauricular subcutaneous plane and is extended postauricularly. Dissection should be accomplished proximal to the facial musculature with careful avoidance of the postauricularis muscle and the great auricular nerve. Dissection is carried into the preplatysmal plane in the neck toward the midline. Effort is made to maximize the medial and inferior extent of the neck dissection from this approach. To complete the preauricular subcutaneous component, sharp dissection is carried to the diagonal line 


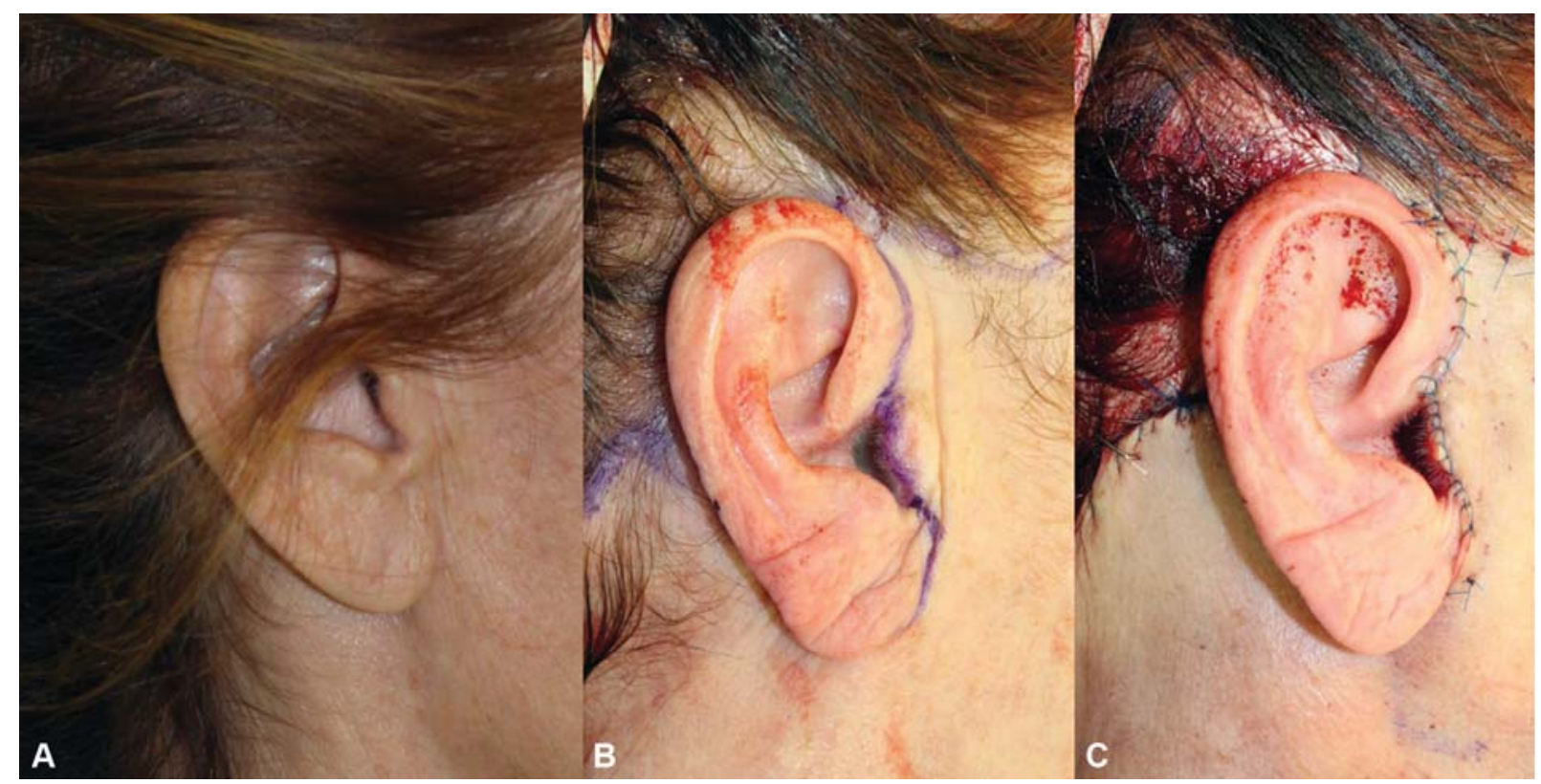

Fig. 7 (A) Preoperative upright photo isolating the ear. (B) Intraoperative appearance of the same patient in supine; note that the earlobe shape and rhytids are significantly different from the preoperative upright photo. (C) Once the incision is made, the intraoperative reference is lost. Care must be taken to duplicate the supine intraoperative earlobe anatomy, not the photo.

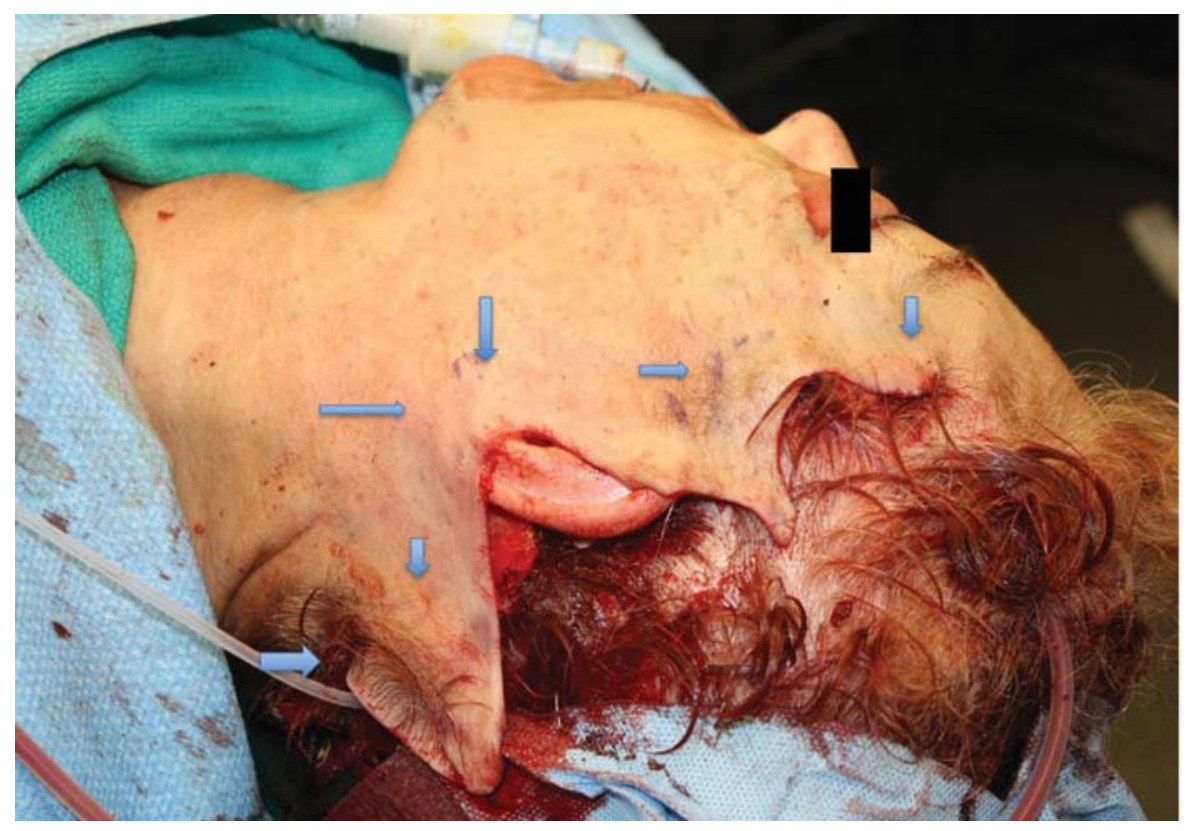

Fig. 8 A 62-year-old female undergoing deep-plane rhytidectomy, demonstrating excessive soft tissue redundancy. Note the temporal incision line, avoiding temporal hair tuft movement during redraping.

extending from the angle of the mandible to the lateral border of the orbicularis oculi muscle ( - Fig. 6 ). The angle of the mandible is used as a reference because it often represents the anterior border of the parotid gland. Since the SMAS and the deep cervical fascia are fused at the parotid, the deep plane cannot be entered until dissection proceeds anterior to the parotid. The fusion of the fascia layers causes the facial soft tissue in the lateral midface to be anchored, whereas the medial soft tissue is mobile. This explains why laterally based SMAS procedures, with limited medial flap dissection, are less effective in mobilizing the midface ( - Fig. 9). Deep-plane entry point will vary subtly based on soft tissue thickness, compliance, and whether the procedure is a revision. The greater the face thickness and/or compliance, the more medial the entry point.Revision cases often have a lateral/preauricular soft tissue deficit and require a more lateral entry point. Variance in flap entry point can also influence facial thickness. The more lateral the superior entry, the greater the soft tissue thickness repositioned. This can be used to further volumize the malar region with the thicker flap acting as a pedicled fat graft.

Safe entry into the deep plane can be achieved by creating reference points and using them in defining less obvious 


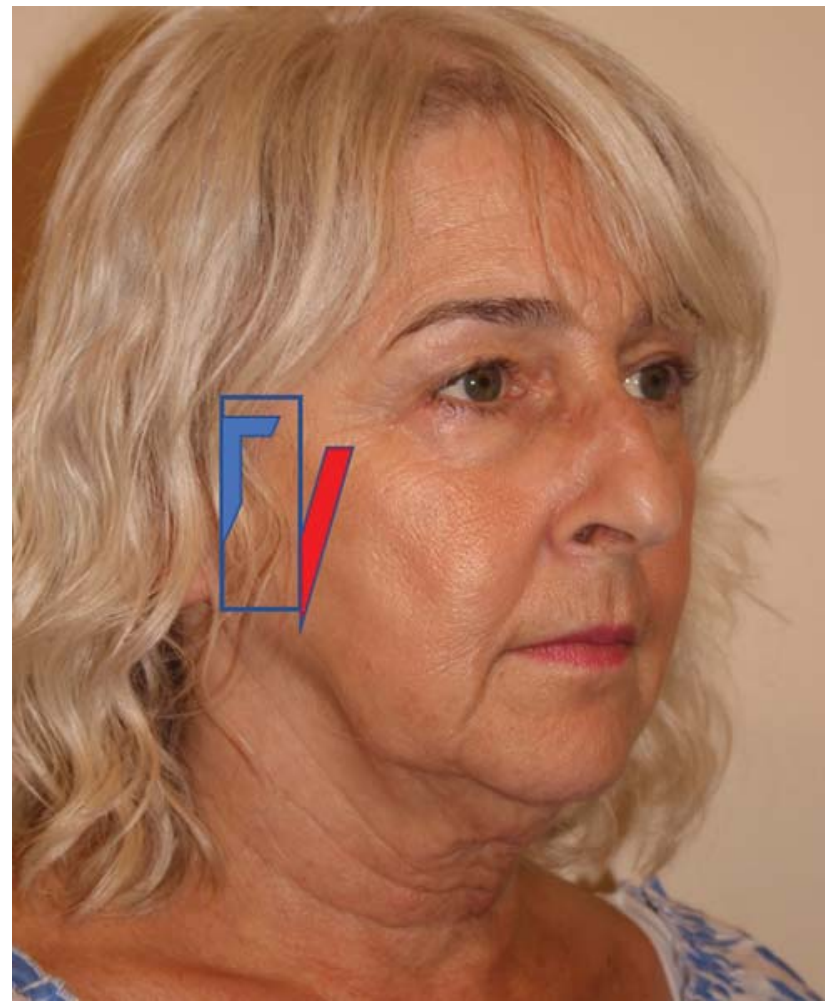

Fig. 9 Understanding the deep-plane advantage through horizontal anatomy. The rectangle shows the fixed parotid and cervical fascia. Note that the minimal aging changes occur laterally. The blue outline shows where superficial muscular aponeurotic layer procedures are accomplished in the immobile lateral face. The majority of midface aging changes are medial to the red tangent, which is the entry point into the mobile deep-plane.

aspects of dissection. The first reference point is established at the lateral border of the orbicularis muscle, inferior to the lateral canthus. In the deep plane, unlike the composite rhytidectomy, the orbicularis oculi muscle is not part of the flap $^{6}$ ( - Fig. 10A, B). Maximal outcomes will best be achieved by taking care to incorporate the majority of the soft tissue into the flap along the superior facial mimetic muscles. Of note, some of the temporal-to-orbicularis dissection can be performed bluntly. The ease of this part of the dissection often foreshadows the ease of the upcoming sub-
SMAS dissection. The deep plane will be entered using a no. 10 blade to create a sharp edge, or shelf, that will be used to mobilize and fixate the complex flap at a later stage in the procedure. Definition of the deep plane should first be accomplished just superior to the angle of the mandible, where the masseter muscle is an obvious deep boundary and the SMAS is well developed and incorporated with the platysma muscle.

Categorizing the patient's face type is integral in predicting plane depth. Thin faces tend to have a thin and poorly developed SMAS/platysma, and the difference in depth between the subcutaneous dissection and the deep plane may be minimal. The platysma muscle will first be encountered as one proceeds deeper in the dissection. Platysma can be recognized by its transverse muscle fibers in contrast with the vertically oriented muscle fibers of the deeper masseter muscle. Once beneath the SMAS, the fibroareolar tissue representing the potential space defining the deep plane becomes evident (-Fig. 11A, B). At this point, it is easy to bluntly dissect the fibroareolar tissue to further establish the deep plane. Often blunt dissection can glide along anteriorly past the masseter muscle to the perioral area with little resistance. Minor masseteric cutaneous ligaments can be encountered, but these are comfortably lysed. If the fibroareolar tissue representing the deep plane is not encountered, an anterior extension of the parotid gland may be inhibiting plane entry. Dissection should proceed medially until the parotid is passed and the fibroareolar tissue representing the deep plane becomes evident.

The second reference point, the masseter muscle, now becomes evident. It is covered by the superficial layer of the deep cervical fascia, which protects the underlying facial nerve. This reference point, along with the lateral border of the already defined orbicularis oculi muscle, permits the deep plane to be developed anteriorly over the masseter muscle and buccal fat pad toward the mouth. Deep-plane development is also carried superiorly to the inferior border of the zygomatic major muscle. The deep plane is usually avascular except around the zygoma and submalar regions. Bleeding may occur from the facial or labial arteries if dissection is traumatic. Since branches of the facial nerve accompany these arteries, monopolar cautery should be
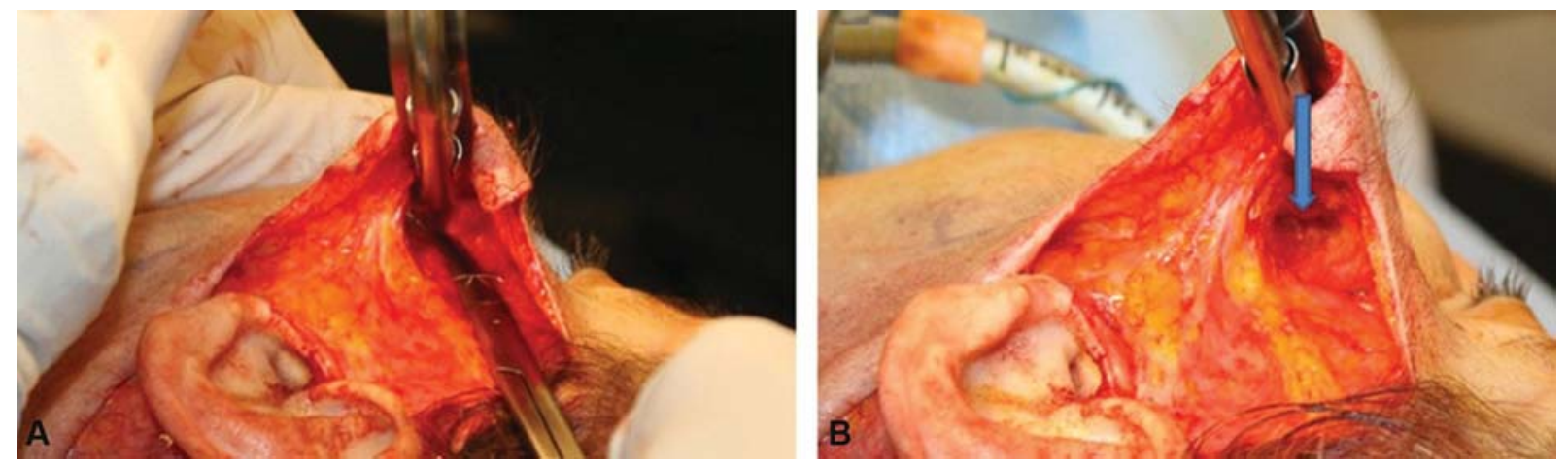

Fig. $10(A, B)$ The initial anatomical landmark to define the deep plane is the orbicularis muscle. Unlike the composite rhytidectomy, the orbicularis muscle is not part of the flap. An arrow indicates the flap being raised. (Reproduced with permission of Gordon NA, Adam SI. The deepplane approach to neck rejuvenation. Facial Plast Surg Clin North Am 2014;22(2):269-284.). 

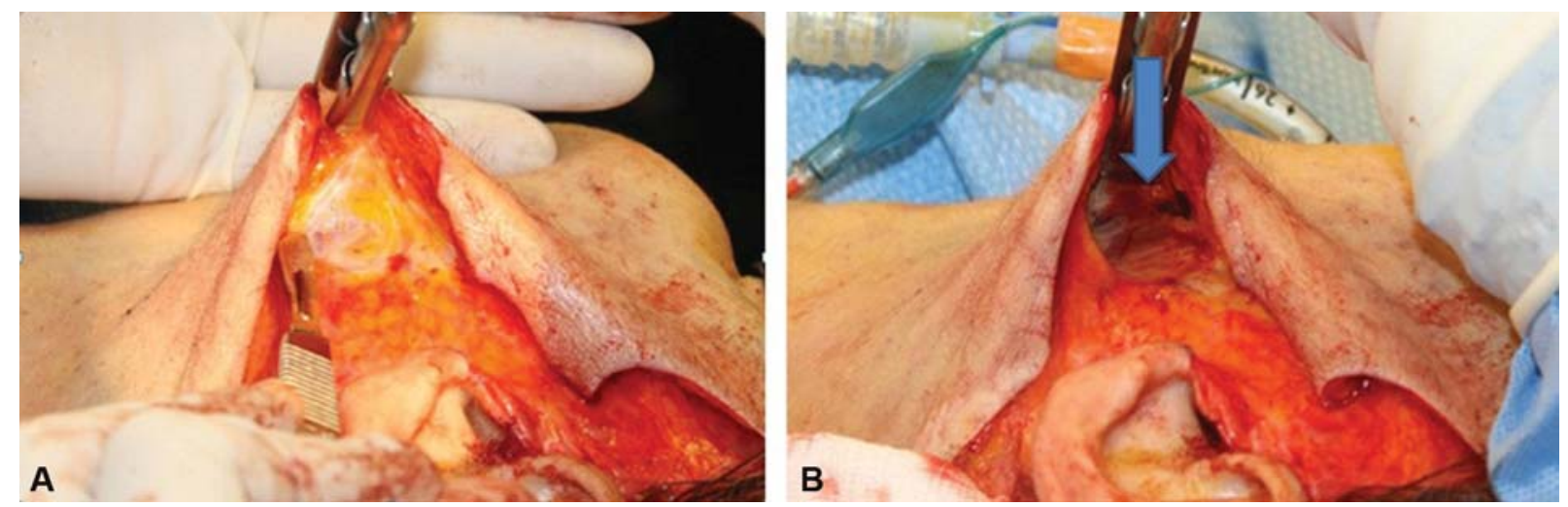

Fig. 11 (A) The establishment of the deep plane just superior to the angle of the mandible. This anatomical landmark is used because the deep plane has a well-defined deep boundary, the masseter muscle, and a well-developed superficial muscular aponeurotic layer/platysma muscle unit. (B) Fibroalveolar tissue defines this potential space. (Reproduced with permission of Gordon NA, Adam SI. The deep-plane approach to neck rejuvenation. Facial Plast Surg Clin North Am 2014;22(2):269-284.)

avoided and simple pressure and hypotensive anesthesia will typically tamponade any bleeding that may be encountered.

At this point in the procedure, the only region yet to be dissected is the bridge of soft tissue inferior to the initial orbicularis dissection and superior to the masseter dissection. Sharp dissection is necessary in this area where the zygomatic cutaneous ligaments will be encountered. Release of these ligaments is necessary to fully mobilize the midface, especially the malar fat pad, and positively influence the nasolabial folds and restore malar width ( $\mathbf{F i g . ~ 1 2 A , ~ B ) . ~}{ }^{14-16}$ These ligaments cannot be lysed bluntly: failure to release them severely limits the extent of midface mobilization and hinders treatment outcome. If the ligaments are not released, the procedure would be defined as a modified deep-plane rhytidectomy. Using the defined inferior and superior plane, one can comfortably cut through this retaining ligament. Once medial, blunt dissection can often be used to continue anteriorly along the zygomatic major muscle. Care is taken during this part of the dissection to incorporate the majority of malar fat pad soft tissue into the flap, leaving a relatively skeletonized zygomatic major muscle. A combination of blunt and sharp dissection is performed anteriorly to the nasolabial fold. Gentle dissection is used when approaching the oral commissure to protect the labial branch of the facial nerve, which becomes superficial and can be injured by overly vigorous dissection. Notably, prior documentation of dimples is predictive of the zygomatic major muscle anatomy. As a classic midface dimple is caused by either a fascial band extending from the zygomatic major muscle (minor dimple) or a frank bifid zygomatic major muscle (major dimple), care must be taken to preserve this offshoot of the zygomatic major muscle as it travels to the dermis inferiorly.

The midface is degloved in the embryologic cleavage plane at this point in the procedure (-Fig. 13). The deep-plane dissection can be extended in several ways and customized to the extent of aging changes, soft tissue type, and neck difficulty. In standard cases, release of the anchoring ligaments at the angle of the mandible will eliminate counterforce pulling the midface inferiomedial, preventing neck failures and extending outcome longevity. In cases of stiff noncompliant soft tissue, revision cases, and difficult neck anatomy, further extension of the deep-plane dissection by releasing cervical ligament will create an even greater platysma muscle and flap mobility, maximizing lateral neck outcomes and longevity. Since the deep plane has been defined with the superficial layer of the deep cervical fascia as a guide, further inferior dissection and
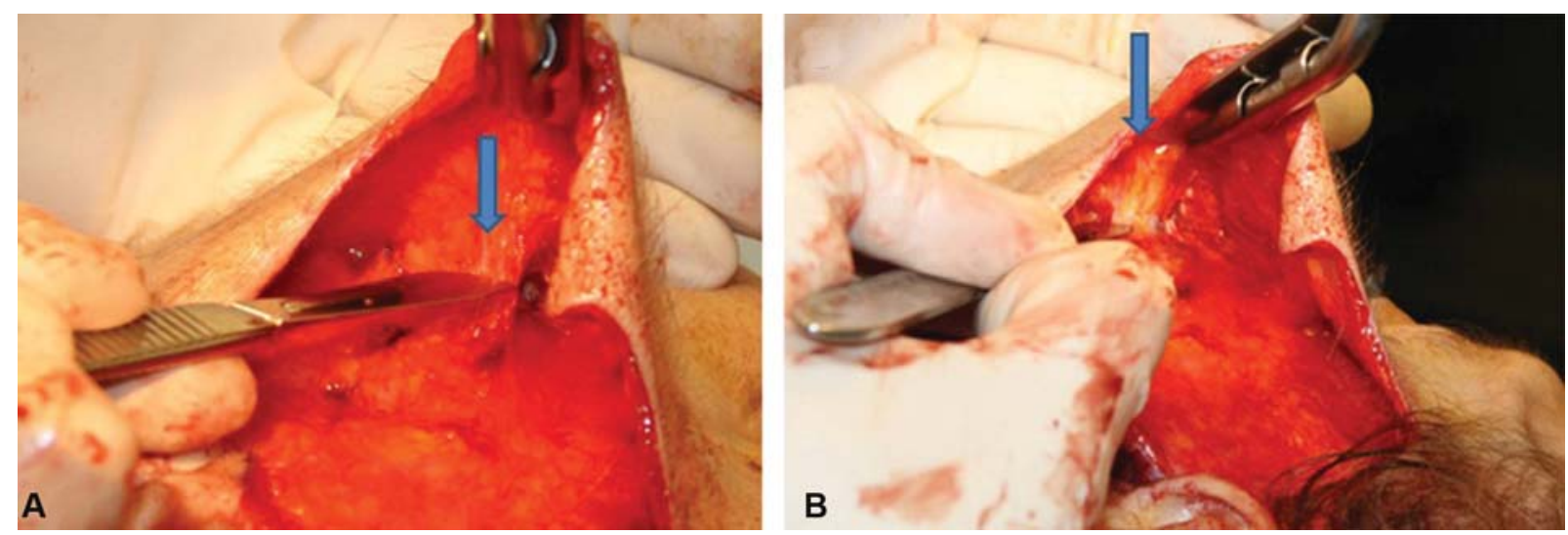

Fig. 12 (A,B) Sharp lysis of the zygomatic cutaneous ligament (arrow). This allows malar fat pad repositioning for the treatment of the nasolabial folds and restores facial width. (Reproduced with permission of Gordon NA, Adam SI. The deep-plane approach to neck rejuvenation. Facial Plast Surg Clin North Am 2014;22(2):269-284.). 


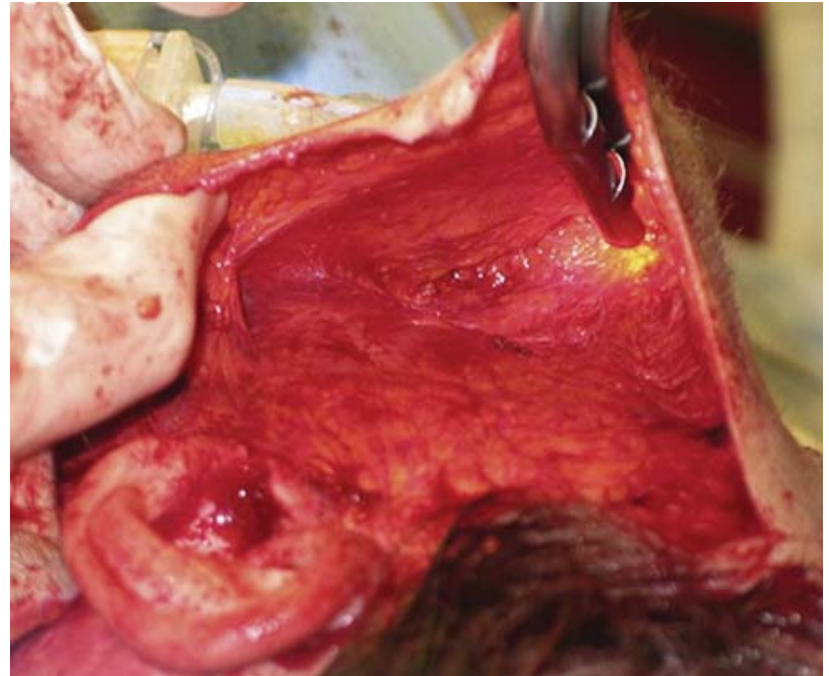

Fig. 13 A fully degloved face in the deep plane. The retractor is tenting the zygomatic major muscle into view. A clear superficial muscular aponeurotic layer/platysma shelf is evident along the deep aspect of the flap (Reproduced with permission of Gordon NA, Adam SI. The deep-plane approach to neck rejuvenation. Facial Plast Surg Clin North Am 2014;22(2):269-284.)

release of the cervical retaining ligaments can be accomplished safely. First, the region between the angle of the mandible and the facial artery can be undermined inferiolaterally below the mandible by blunt dissection. Although this is usually a nontraumatic dissection, the marginal mandibular nerve can be stretched in stiff faces and certain revision cases.

Since the lower midface (at the angle of mandible) and the posterior platysma muscle (at the sternocleidomastoid muscle) are fixated by the cervical retaining ligaments, partialto-full release of the ligaments is a case-specific graduated approach to further mobilize the midface and, to a greater extent, the lateral platysma muscle neck complex. Using the previously dissected deep plane at the midface as a guide, the midface can be retracted to define the posterior border of the platysma muscle between the great auricular nerve and the external jugular vein. Sharp dissection from the angle of the mandible with a no. 10 blade proceeding inferiorly along the posterior platysma to the level of the superficial layer of the deep cervical fascia begins the ligament release. Dissection in this plane can continue medially using scissors conservatively by spreading or continue with a no. 10 blade. The ligaments continue medially but only have to be released to the desired mobility. In difficult neck anatomy such as ptotic submandibular glands, a back-cut can be incorporated in the platysma muscle ( $\mathbf{- F i g}$. 14). This can be performed two- to three-finger breadths below and parallel to the mandible toward the midline and can extend as far medially as the submandibular capsule. This platysma back-cut can be rotated and fixated superiolaterally toward the mastoid to provide additional support for the lateral neck structures. Since transections of the platysma can lead to permanent ridging and tight neck syndrome, we reserve this for only more severe cases. An additional advantage of extended release is more complete exposure of the parotid gland and a view into the lateral neck subplatysmal anatomy (-Fig. 15).

After degloving the midface in the deep plane, the deep facial structures contributing to aging, such as the buccal fat pad, can be individually assessed. The buccal fat pad is covered by the superficial layer of the deep cervical fascia. As the facial skeleton subtly shrinks, the fascia can become weak or ptotic and cause the buccal fat pad to pseudoherniate. This process is similar to the concept of orbital fat pseudoherniation that contributes to ocular aging changes. The pseudoherniation of the buccal fat pad ${ }^{17,18}$ can contribute to jowling or even a "cheeky" appearance (-Fig. 16A) and can be individually assessed in vivo from the deep-plane dissection. Since the patient is supine intraoperatively, a

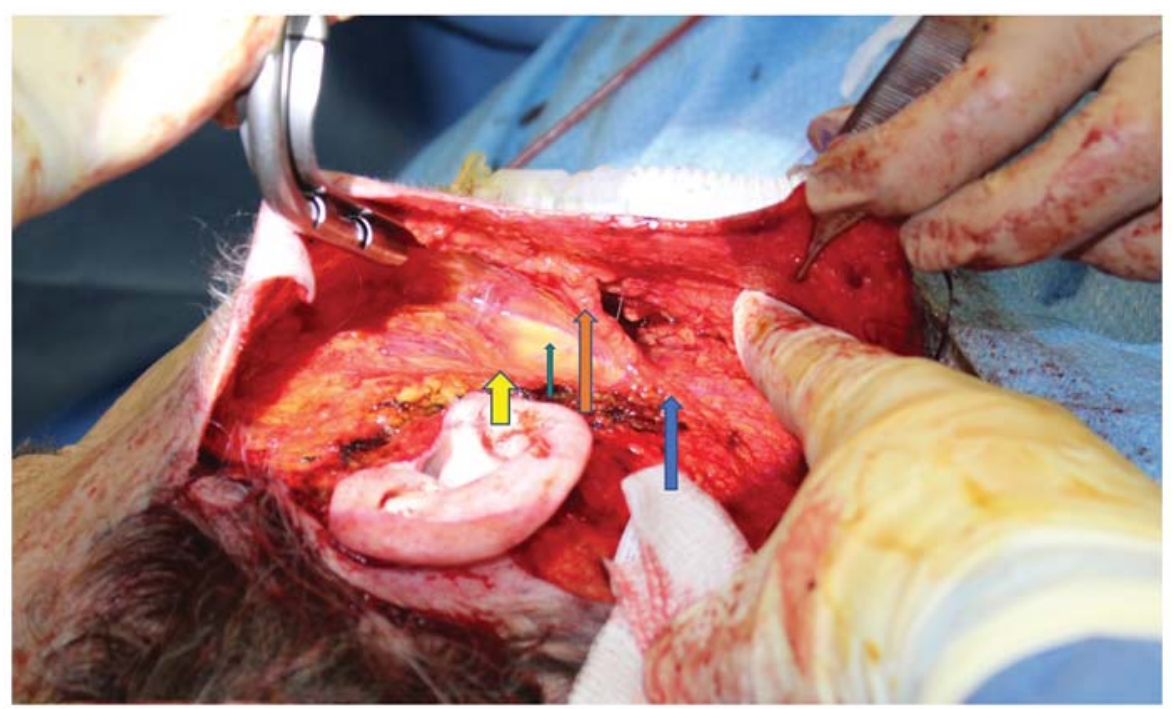

Fig. 14 Extended deep-plane dissection. Yellow arrow shows the angle of the mandible, original inferior limit of the deep-plane dissection. Blue arrow shows the inferior extent of the cervical ligament release. Orange arrow shows the area where platysma back-cut would be performed. Thin green arrow reveals subplatysmal fat. Release of cervical ligaments and inferior extension of deep plane maximizes neck outcomes and longevity. 

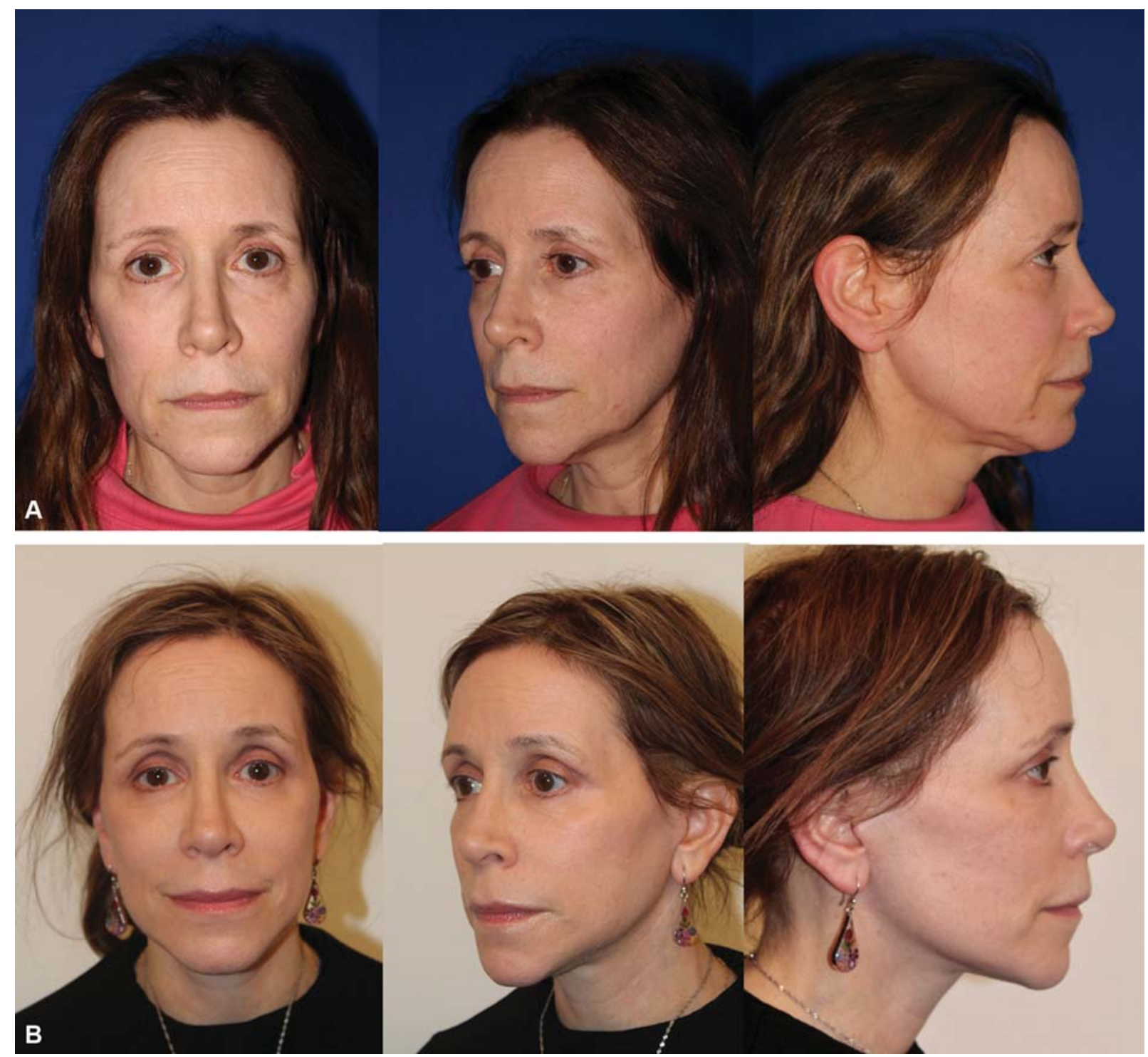

Fig. 15 (A) Preoperative photographs of a 58-year-old woman with moderate aging changes. (B) Postoperative photographs after extended deep-plane rhytidectomy, endo/coronal forehead lift, lower blepharoplasty, and periocular and perioral laser skin resurfacing with erbium/yttrium aluminum garnet laser. No platysma back-cut or buccal fat was removed. Note the ability of the procedure to be tailored based on in vivo anatomy assessment.

fullness or bulge of the buccal fat pad will understate its contribution to a jowl and should be correlated with preoperative photos to make a precise judgment regarding the amount and position of buccal fat excision (-Fig. 16B). If buccal fat pseudoherniaton is present, we will make a small nick incision through the deep cervical fascia using sharp scissors. The surgical assistant applies pressure on the buccal fat pad while a conservative amount of buccal fat is gently teased through the nick incision and resected using bipolar cautery, with strict observation for any facial twitches ( - Fig. 17A, B). The contour of the buccal fat pad immediately becomes more linear, often after excision of only a small amount of buccal fat. If too much buccal fat herniates through the nick incision, then it should be gently placed back into the subfascial pocket. Overaggressive buccal fat resection can create an unnatural perioral facial crease. This last maneuver completes the midface dissection, which is repeated on the contralateral side (-Fig. 18).
To complete the neck dissection, a horizontal incision is made either in the submental crease or slightly inferior to it. Defining superficial neck issues from deep neck aging changes is paramount to success and avoiding a "cobra deformity" complication. Preoperative photos are referenced to define the pattern, symmetry and degree of platysma banding, and fat deposition, and to evaluate the submandibular/digastric triangle. Where superficial neck aging changes are predominantly medial and can be significantly addressed by midface mobilization, deep neck aging is paramedian and must be approached directly. The digastric muscle can often be differentiated from the platysma by following the outlines of the muscle and its relationship to the hyoid. The anterior digastric muscle extends to the lateral aspect of the hyoid bone and then redirects superolaterally in contrast to the more medial platysma that will cross and even obscure the hyoid (-Fig. 19). This distinction is important to guide further neck treatment. 

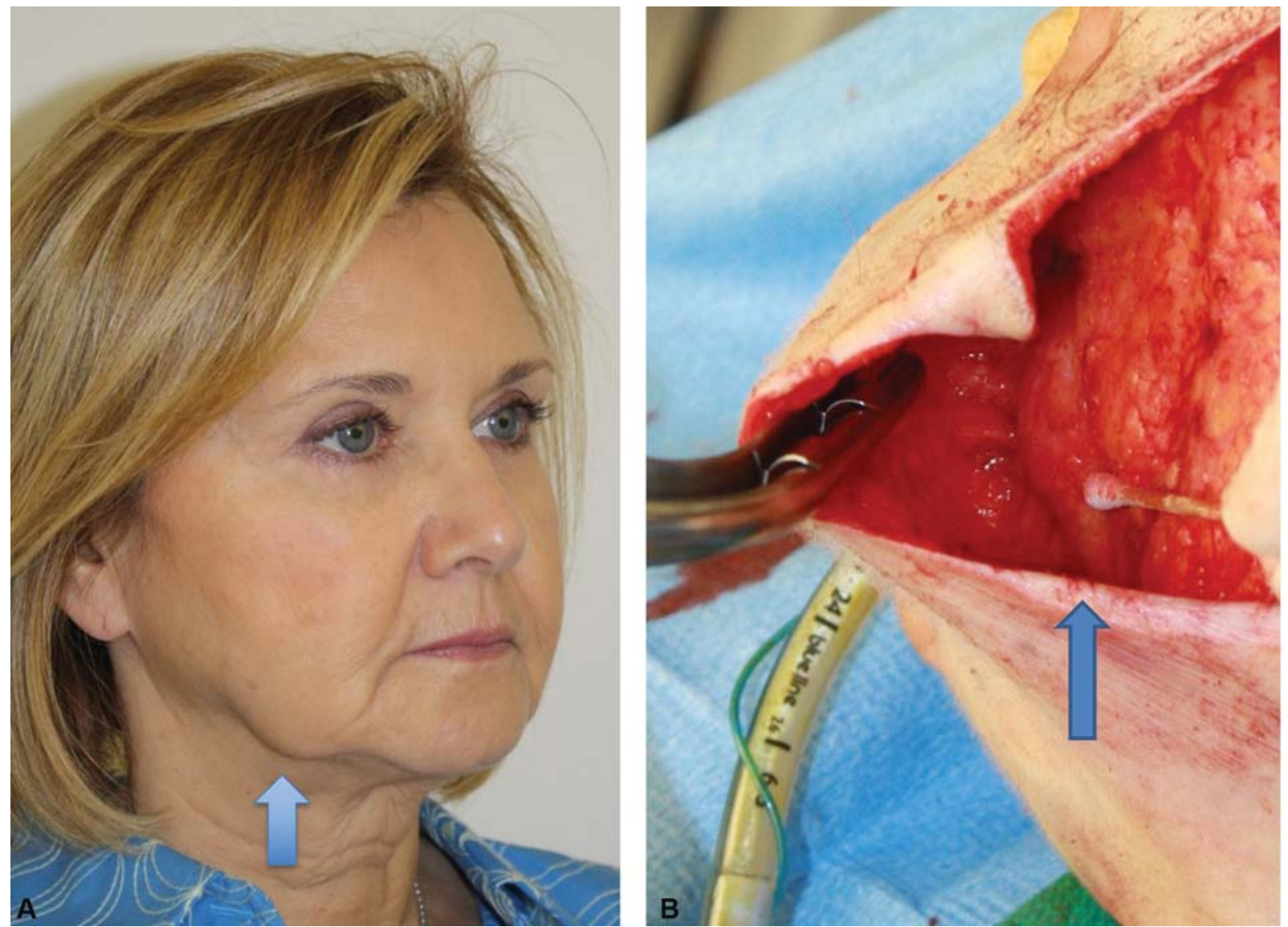

Fig. 16 (A) Preoperative photo correlating the external appearance of a jowl with (B) an intraoperative photograph revealing buccal fat pseudoherniation in the same position. This confirms the pseudoherniated buccal fat contribution to jowling. Of note, the intraoperative photograph was taken supine and rotated for comparison. This underestimates the amount of actual pseudoherniation.
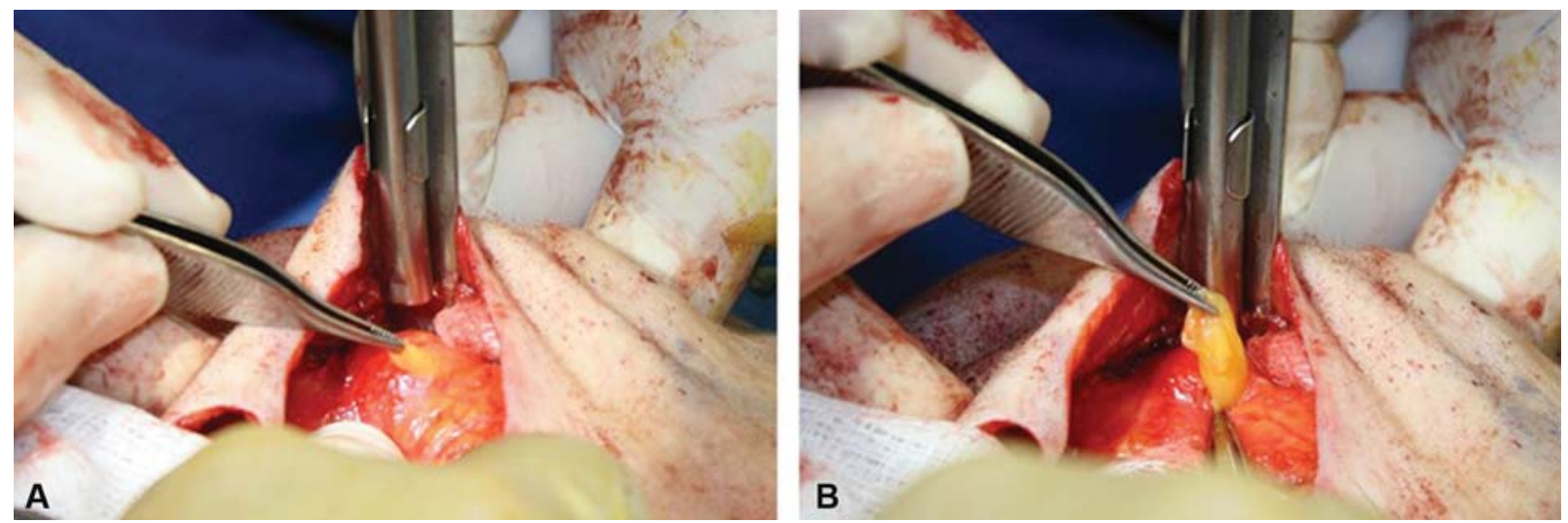

Fig. 17 (A, B) Mild pressure is applied to the buccal fat pad by the surgical assistant as a nick incision is made into the fascia, allowing precise removal of buccal fat. (Reproduced with permission of Gordon NA, Adam SI. The deep-plane approach to neck rejuvenation. Facial Plast Surg Clin North Am 2014;22(2):269-284.)

Both subplatysmal fat and ptotic submandibular glands are often seen as paramedian fullness.

After incision, the subsequent preplatysmal dissection will connect the submental dissection to the completed bilateral facial dissections. Care is taken to only grasp the redundant platysma muscle in the midline. After crossclamping, redundant muscle is resected. The next steps taken will be defined by the assessment of the subplatysmal fat and digastric/submandibular gland component previously eval- uated. Since many surgeons may not feel comfortable treating lateral subplatysmal structures (fat, digastric, and submandibular gland), it is important to not overtreat the midline structures, platysma, and pre- and subplatysma fat disproportionately to the lateral deep neck structures or else a "cobra deformity" will be created. Dissection proceeds in the subplatysmal plane until the capsule of the submandibular gland is exposed. Partial resection of the inferior aspect of the anterior belly of the digastric near the hyoid is an 

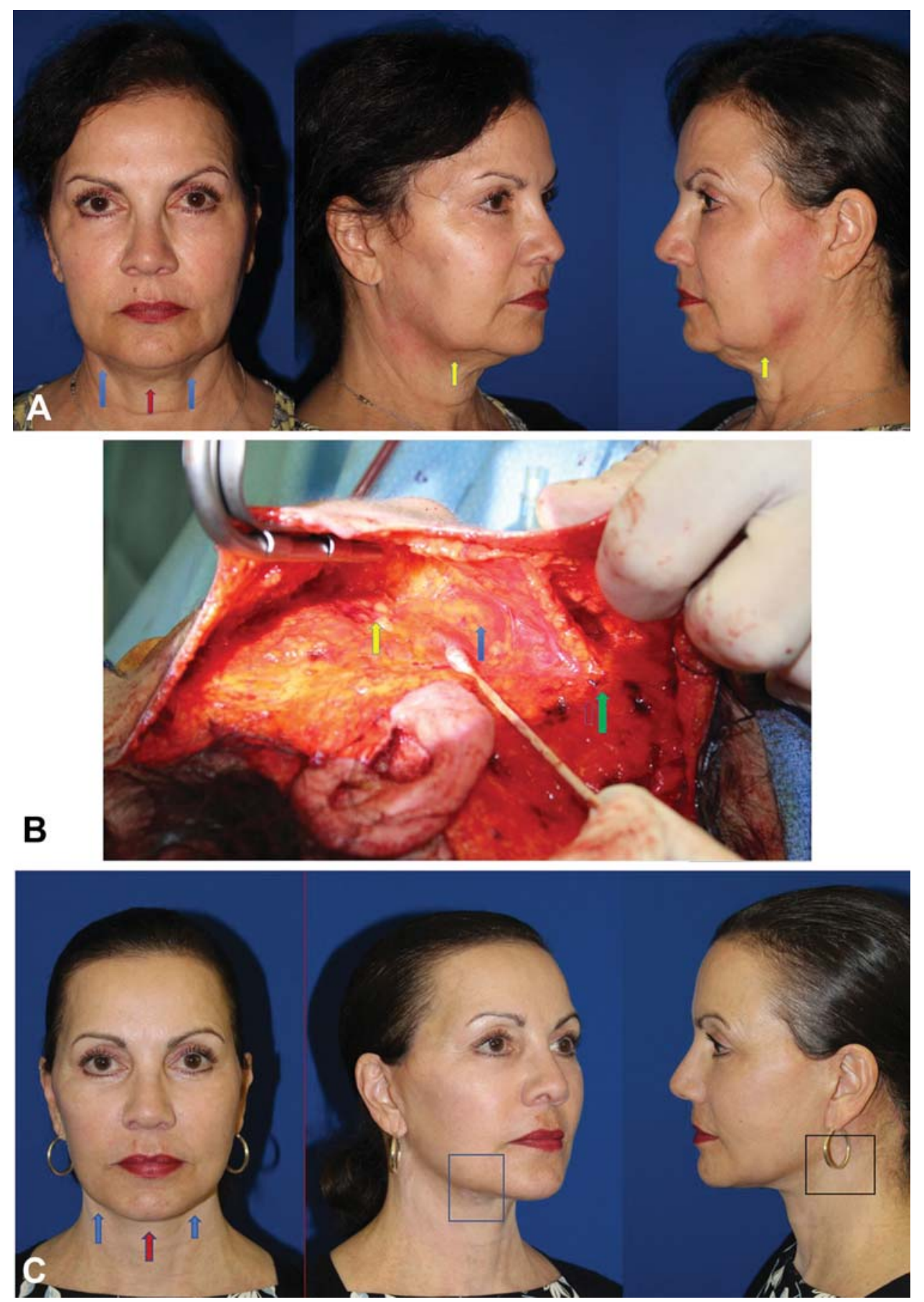

Fig. 18 (A) Preoperative photograph of a 58-year-old woman; anatomically accurate diagnosis of both superficial and deep aspects of facial aging is imperative to create an comprehensive rejuvenation. Red arrows represent the superficial/medial neck. Blue arrows represent the paramedian/deep subplatysmal neck structures. Yellow arrows represent potential pseudoherniated buccal fat. (B) Intraoperative photograph showing case-specific dissection and treatment. Note the cotton tip applicator on the angle of the mandible. Yellow arrow reveals resected buccal fat pseudoherniation. Blue arrow reveals subplatysmal fat that is soon to be treated. Green arrow shows the extent of inferior cervical ligament release. (C) Postoperative photographs after undergoing extended deep-plane rhytidectomy with buccal fat removal, endo/coronal forehead lift, lower blepharoplasty, and periocular and perioral erbium/yttrium aluminum garnet laser skin resurfacing. Note that the extended deep-plane procedure allows an in vivo assessed, anatomically correct rejuvenation. Red arrow shows the superficial/ medial neck. Blue arrows show the paramedian/deep neck. Blue rectangle shows the medial jawline. Black rectangle shows the lateral jawline.

option for the experienced surgeon. Although near-total resection is well described, we perform a limited submandibular gland resection, when indicated, by opening the medial capsule and only resecting the herniated gland by bipolar or suction cautery. Our conservative approach is based on issues related to incomplete gland removal, salivary leaks, potential late arterial bleeding, risks to the marginal mandibular nerve, and prolonged recovery. ${ }^{19}$ Subplatysmal fat can be resected between the digastric muscles in individuals with significant subplatysmal fatty deposition. The 


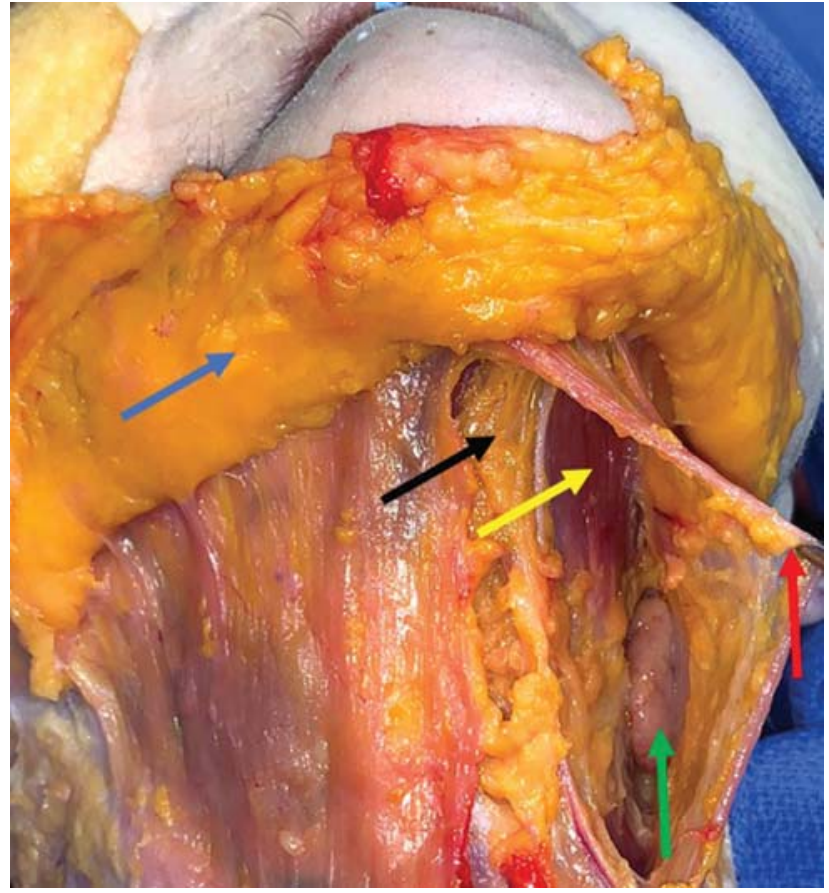

Fig. 19 Cadaveric dissection. Red arrow shows the retracted platysma, green arrow shows the submandibular gland, yellow arrow shows the anterior digastric muscle, black arrow shows the subplatysma fat, and blue arrow shows the supraplatysma fat. Note the more lateral position of the digastric muscle compared with the more medial extension of the platysma

(Reproduced with permission of Charafeddine AH, Couto RA, Zins JE. Neck rejuvenation: anatomy and technique. Clin Plast Surg 46(4):573-586.)

subplatysmal region tends to be arterial and vascular, and therefore be prepared to address bleeding. We follow a more conservative approach when the digastric/submandibular triangle is ptotic and not being treated, as excessive subplatysmal fat resection can enhance and/or expose these lateral neck aesthetic issues. In addition, overzealous treatment of the deep neck, especially in patients with a long neck and/or thick soft tissue, can lead to a skeletonized or "neck dissection" appearance.

Once any subplatysmal fat excision is completed, the previously resected redundant platysma muscle is imbricated using buried Prolene sutures (Ethicon); 4-0 Prolene is often used proximal to the mentum, whereas 3-0 Prolene sutures are used until the hyoid bone is encountered. Imbrication of the platysma should not proceed below the hyoid to prevent bowstringing of the muscle. One small finger should fit in the gap between the muscle edge and the hyoid at completion of the imbrication. In patients with extended platysma banding, an anterior strip of the platysma inferior to the last fixation suture can also be resected. It should be noted that even in severely ptotic neck contours, significant platysma muscle resection may not be needed.

Since the majority of the neck contour will be recreated by the vertical suspension of the platysma/SMAS in the midface during the upcoming flap fixation, vertically lifting the midface intraoperatively will show the final neck contour and reveal any untreated preplatysmal fat and assess the need for mandibular ligament release.
Preplatysmal fat contouring completes the neck component. Most fat contouring is performed submentally by a sharp, direct dissection in this region. Care is taken to keep an even layer of fat on the skin flap, preventing irregularities and dermal-platysmal adherence. Fat removal should be very conservative, or avoided, in necks with poor skin elasticity confirmed by deep horizontal rhytids. Repeated vertical lifting of the midface will show the completed neck contour and reveal any irregularities that need further contouring. If accessible, running your fingers along the underside of the skin flap will reveal specific areas in need of contouring.

Unlike the release of deep zygomatic cutaneous ligament necessary for mobility of the entire cheek soft tissue, mandibular ligament release is accomplished superficially and has limited significance. When we are skeletonizing the inferior edge of the mandible with sharp subcutaneous dissection, continuation superiorly over the easily defined ligament causes release of the ligament; since it is vascular, it usually requires cauterization. We use this release in revision cases, stiff soft tissue, or significant marionette lines. The neck is now copiously irrigated with multiple cool saline washes and one betadine wash to minimize residual necrotic fat cells that may serve as a future nidus of infection. Cautery is avoided on the skin flap to prevent delayed skin necrosis. The submental incision is now closed with 5-0 Prolene sutures.

Now flap mobilization, fixation, and closure proceed. After initial hemostasis is achieved in the neck and postauricular region through the use of bipolar cautery, a small bulb drain is placed through a postauricular hairline stab incision. Since the thicker, deep-plane complex flap will be advanced and sutured proximal to the preauricular incision, casespecific preauricular soft tissue contouring and resection is necessary. The new depth created should be consistent with the thickness of the deep-plane flap that will be inlaid. This process ensures that the bulkiness of the complex flap does not result in unnatural alterations of the normal preauricular and tragal architecture. Subcutaneous contouring can be used to prevent facial widening. It is important to note that subcutaneous tissue should only be resected to the parotid fascia, which is necessary to keep intact for anchoring the advanced flap. Contouring is not usually necessary superior to the tragus as the soft tissue addition from the thicker flap will produce subtle widening in the malar region. In fact, during the creation of the deep-plane flap superiorly, a more lateral entry, lateral to the orbicularis oculi muscle, can be designed to transfer a thicker flap to the lateral malar area; this produces the effects of fat grafting, widening, and volumizing the upper lateral midface.

Preauricular contouring can also be used to create a more tapered lower midface, especially in patients with prominent parotid glands ( - Fig. 20A, B). Once the parotid fascia is reached by sharp resection, bipolar cautery is used to shrink the parotid from the tragus inferiorly to the sternocleidomastoid muscle and anteriorly over the masseter muscle. Great care and operative staff facial observation is used to avoid stimulating the facial nerve. Significant shrinking, 

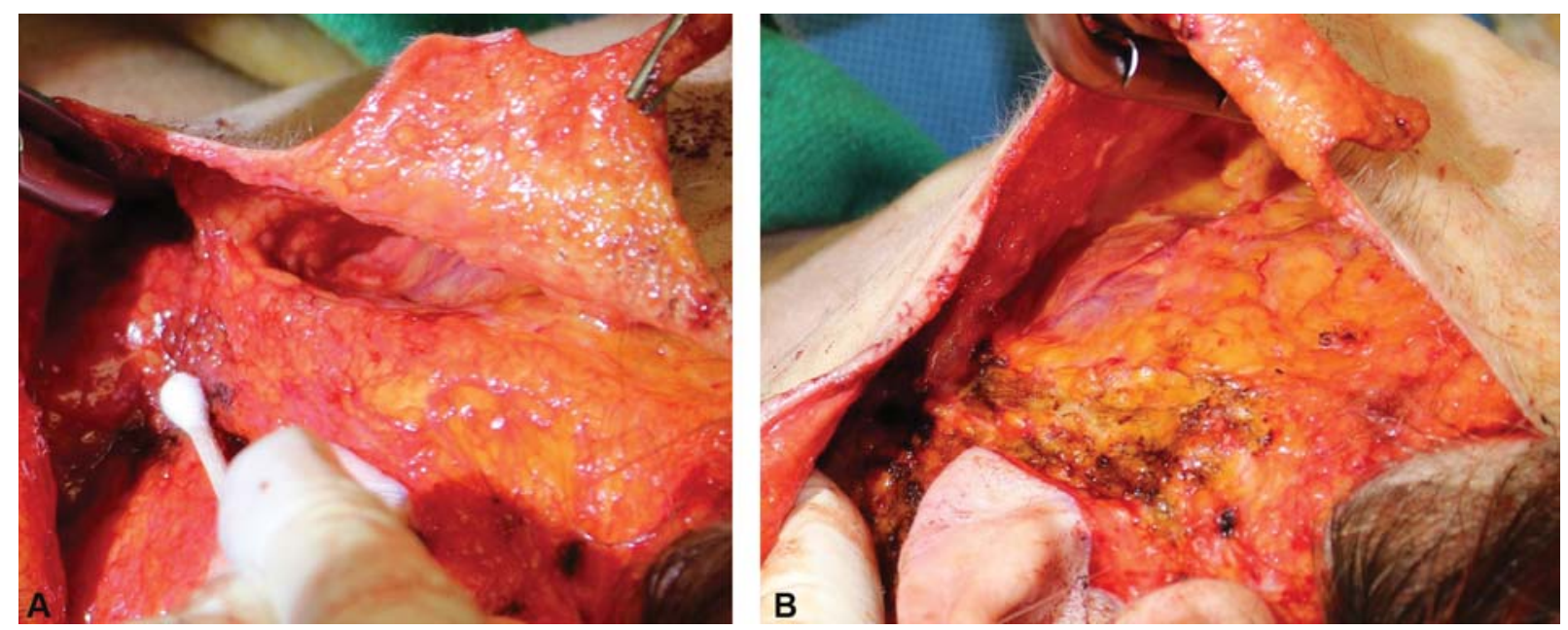

Fig. 20 (A) Intraoperative view of the preauricular region after flap dissection showing thick soft tissue contributing to lower face widening with age. (B) View after contouring of both the soft tissue and hypertrophic parotid, creating lower midface narrowing.

narrowing, and sculpting can be achieved by this technique. A betadine-soaked sponge is applied to prevent infection from fat necrosis when bipolar is aggressively applied.

Although emphasis is placed on dissection, it is nuances of flap advancement that are critical in maximizing results and avoiding potential issues. Since the platysma/SMAS is now a sling from the submental region through the midface, the goal is to mobilize and fixate the complex flap in the most vertical vector as other factors will allow. Although the vector is never truly vertical, and often parallels the vector of the zygomatic major muscle, the degree and longevity of outcome, both in the midface and neck contour, is directly related to vertical suspension. Variables such as degree of soft tissue redundancy, compliance, temporal hair patterns, concomitant forehead treatment, and whether the case is a primary or revision rhytidectomy also affect flap vector planning.

In general, in patients with compliant, overly redundant soft tissue, the soft tissue will mobilize to such a great extent that it will transfer to the temporal region and create "dog ears" unless extended incisions are used along the entire temporal hair tuft (-Fig. 8). In extreme cases, vertical tissue transfer superior to the lateral canthus can cause an unnatural rotation in the relaxed skin tension lines and should be avoided. Creating a more posterior vector by angling the flap at the helix can help avoid these issues. Soft tissue bunching can also occur if the rhytidectomy is not combined with a browlift. In patients with limited temporal hairlines, such as men, an anterior extension incision can be placed from the temporal hair tuft toward the lateral canthus in a relaxed skin tension line to avoid this dilemma (-Fig. 21A, B). This is usually reserved for older patients with appropriate rhytids, especially men. These issues emphasize the need for careful planning.

The opposite issue presents in the overresected revision patient. In this case, there is a soft tissue deficit preauricularly in the horizontal plane, and a significant vertical vector cannot be used. Posteriorly based flap advancement is necessary to close the soft tissue defect and remove skin tension from the previous rhytidectomy. Mandibular ligament release is also often necessary to maximize soft tissue mobilization. These cases are more likely to result in "neck failures,"
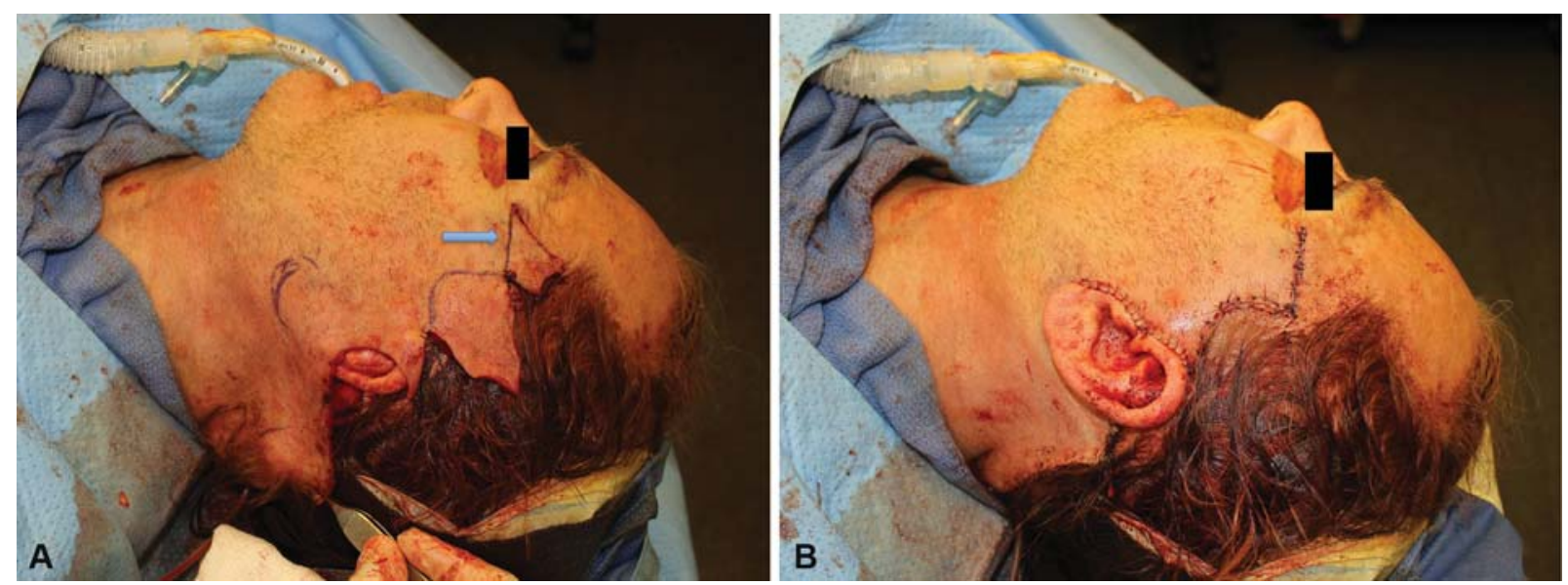

Fig. 21 (A) Intraoperative view of planning of anterior incision extensions (arrow) in a deep plane rhytidectomy case without concomitant forehead lift. Vertical soft tissue mobilization can be maximized and the resultant "dog ear" eliminated. (B) Meticulous planning and suturing allow for cosmetically inconspicuous closure. 

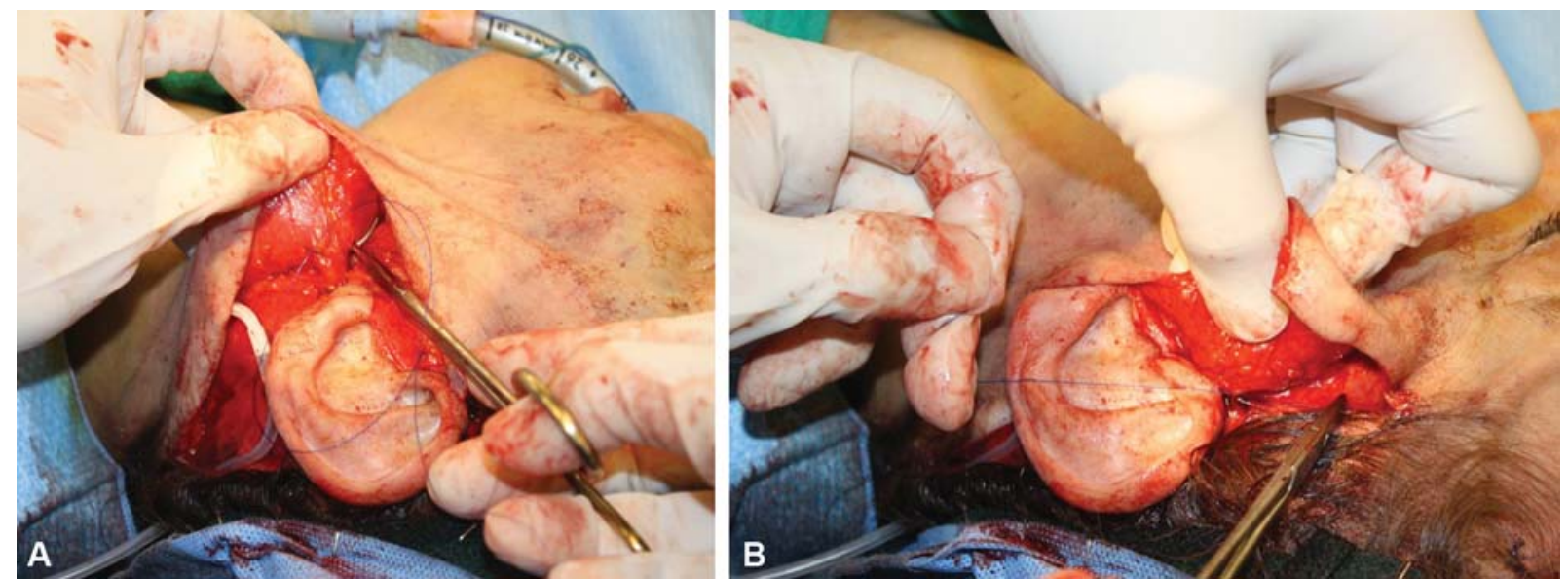

Fig. 22 Flap advancement proceeding from inferior to superior using 3-0 Prolene sutures. (A) The superficial muscular aponeurotic layer/platysma originating at the angle of the mandible can typically be advanced proximate to the earlobe. (B) Tension should be placed on this layer. Note that the variations in soft tissue thickness and patient conditions, such as nicotine use, will vary the desired flap placement.

supporting the necessity of vertical suspension and complete ligament release for optimal neck results.

Combining vector issues and soft tissue types with the location of posterior-based periauricular incisions, flap advancement proceeds with a vertical/posterior vector from inferior to superior using 3-0 Prolene sutures ( - Fig. 22A, B). Commonly, the SMAS fascia originating at the angle of the mandible can be advanced proximate to the earlobe. Subtle differences in the placement of the sutures will also depend on variables such as flap thickness and soft tissue compliance. In general, we choose to place flap advancement sutures buried and as lateral as possible to eliminate significant transition issues that can occur from the midface to the upper face. Soft tissue is further contoured as necessary, including resecting redundant flap thickness to recreate normal periauricular aesthetic architecture. The most superior suture of the advancement flap can be a $4-0$ Prolene suture if the SMAS edge is thin. If cervical retaining ligaments have been released, inferior-based sutures are used to suspend the posterior platysma muscle to the mastoid region. It is imperative to resuspend the platysma after cervical ligament release. Otherwise, the platysma will actually be pulled forward, negatively affecting the neck contour.

Maximal tension, evidenced by the beginning of dermal dimpling, is usually created at the level of the advancement flap fascia. This can be adjusted to the goals and recovery period defined. Importantly, minimal-to-no tension should be created at the skin level for the rest of the closure. A no. 11 blade is used to accurately tack the flap at the superior aspect of the helix as it connects to the temporal incision with 5-0 Prolene suture, which is used for the rest of the skin closure. The final extent of the anterior temporal skin incision is completed based on the avoidance of soft tissue bunching or formation of "dog ears." Meticulous soft tissue tailoring using supercut scissors and a no. 15 blade will provide sharp, crisp edges. Mattress sutures are used at the edge of hairlines. Closure continues postauricularly in a similar fashion, using a tacking suture to match up the posterior hairline. The extent of the posterior incision is also completed inferiorly, again based on avoiding soft tissue bunching or "dog ears." When longer incisions are necessary, a subcutaneous and interrupted $3-0$ or $4-0$ absorbable suture is used in the thicker inferior aspect of the hairline at the nape of the neck to prevent spreading of the incision. Using preoperative supine observation and photos as reference, precise soft tissue tailoring is performed to define the earlobe and maintain tragal shape and thickness. Continued meticulousness will recreate other innate details and is necessary to produce a tension-free preauricular skin closure allowing imperceptible incision lines (-Fig. 23).

As we have defined, with more significant soft tissue mobilization, there is a greater need for extended incisions both temporally and postauricularly. If a limited incision pattern is desired, such as eliminating the postauricular incision component, then more limited deep-plane dissections can be performed in concert with limited flap advancement and fixation. Care in planning is necessary to avoid soft tissue bunching. Of course, limited incision patterns also place constraints on the overall outcome. Once the procedure is completed, topical antibiotic ointment is applied to all incisions and a compression dressing is placed. This dressing is released in the anterior neck after the initial recovery room period prior to discharge. If neck drains are present, they are placed on bulb suction. Blood pressure spikes are avoided during anesthesia emergence and extubation.

\section{Aftercare}

The compression dressing is removed on the morning of postoperative day 1 . Often, two small drains are used in the neck in deep-plane face-lift procedures and are removed with the dressing. Incision lines are gently cleaned with hydrogen peroxide, and topical antibiotic ointment is applied three times daily. Any visible sutures, such as those in the temporal hairline, submental, and preauricular regions, 

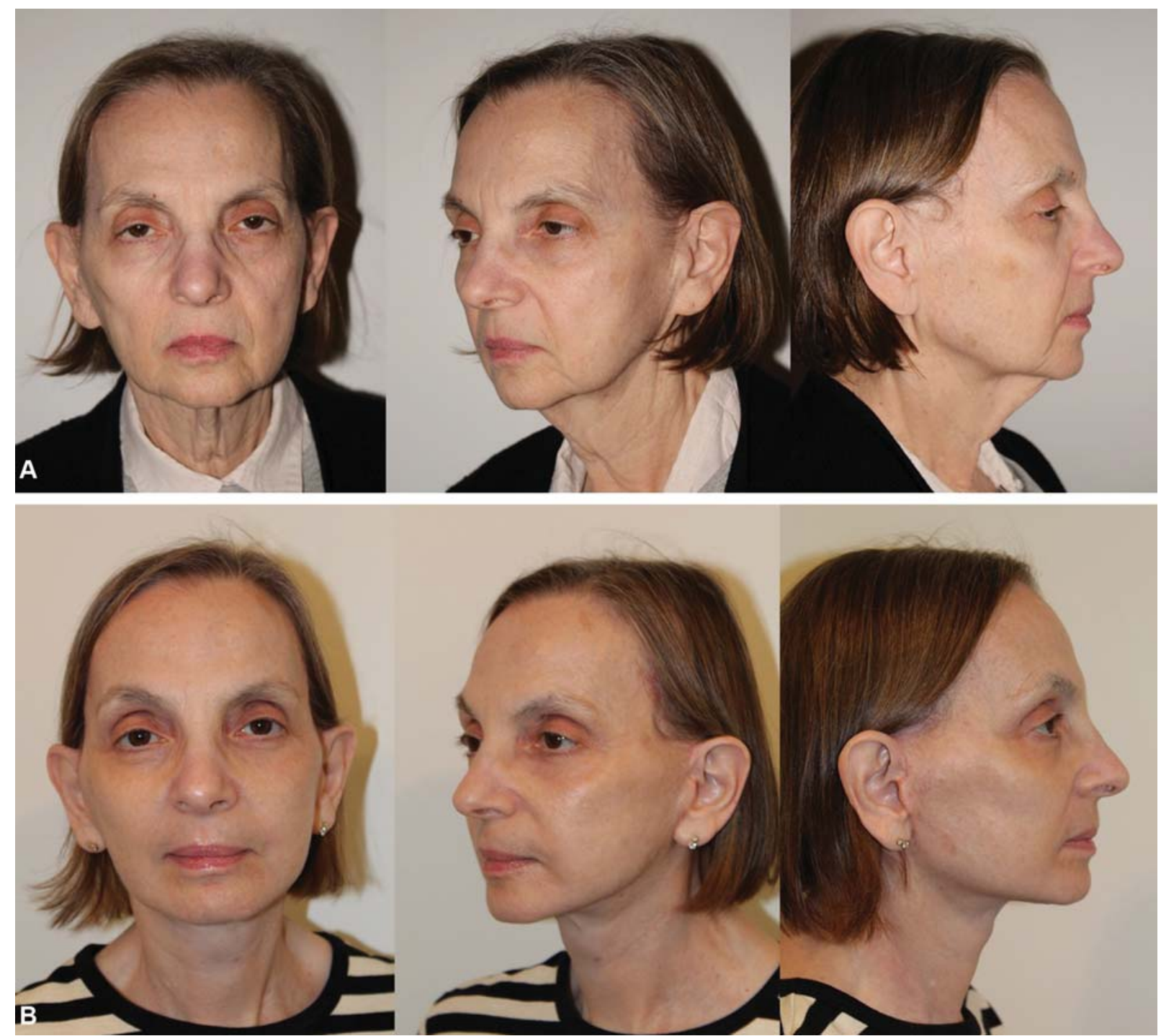

Fig. 23 (A) Preoperative photograph of a 69-year-old woman with significant superficial soft tissue envelope changes and poor hairline. Flap management is essential to manage the excessive soft tissue redundancy within the hairline limitations. (B) Postoperative photographs after extended deep-plane rhytidectomy, endo/coronal forehead lift, lower blepharoplasty, and periocular and perioral laser skin resurfacing. Note the midface volumization with lower face narrowing achieved, whereas a tension-free closure allows hairline incisions and maintenance of earlobe and tragal shape.

are removed on postoperative day 4 . The majority of the remaining sutures are removed on postoperative day 7 . Occasionally, temporal hairline or postauricular sutures are retained for a few more days. Incision lines are sealed with colloidal (or other tissue sealant) and paper tape until they are subjectively defined as mature. Topical scar solution is then applied daily for 2 weeks. Activity is restricted for the first 2 weeks, with general guidelines given to keep the head elevated above the heart level and the heart rate/blood pressure within their relative normal ranges.

\section{Complications}

The complex flap created by the deep-plane dissection is well vascularized, and this makes it resistant to many rhytidectomy complications, ${ }^{20,21}$ especially in marginal patients such as smokers ${ }^{22}$ or diabetics. The rate of hematoma formation is reported to be as low as $1.22 \%$ and is usually in the neck. ${ }^{21,23}$ Infections are also uncommon, reported at $0.73 \%$ of cases, ${ }^{21}$ and are usually limited to the nondeep-plane areas such as the neck. In 17 years, we have never seen an infection in the midface. Skin slough/necrosis rates are similarly low, reported in $0.49 \%$ of cases $^{21}$ and, in our experience, are also limited to nondeep-plane portions of the dissection. The facial nerve injury rate is $0.69 \%,{ }^{21}$ similar to standard facelifts, and has not occurred in our experience.

\section{Conclusion}

The deep-plane technique was originally described by Hamra $^{6,7}$ as an advance of Skoog's ${ }^{4}$ rhytidectomy. The main points in the description were that dissection in the embryologic cleavage plane of the face from the orbicularis oculi to the angle of the mandible allowed both release of the zygomatic 
cutaneous ligament and maximum traction of the complex flap without associated skin traction. However, deep-plane approach has many advantages that were not originally described. Further technical advancements have been accomplished over the past 25 years exclusively using this technique.

First, the access provided by the dissection allows in vivo assessment and treatment of deeper aging structures, such as pseudoherniation of the buccal fat pad and its contribution to jowling. Second, inferior extension of the deep-plane dissections, combined with controlled release of cervical facial ligaments, allows for a more comprehensive treatment of many aging issues. Third, since the flap contains the majority of the facial fat, a larger amount of ptotic soft tissue can be repositioned. Fourth, since the majority of superficial neck aging changes are due to a ptotic superficial soft tissue envelope, "vertical" resuspension of the platysma/SMAS complex not only best reproduces the midfacial contour but also accounts for the majority of the recreation of the neck and jawline. Fifth, exposure of the parotid gland allows in vivo assessment and treatment of parotid gland hypertrophy. Sixth, the resuspension of the posterior platysma, with or without a platysma back-cut, allows greater lateral neck support, contouring, and longevity of the outcome. Seventh, the versatility in design of this well-vascularized flap allows case-specific enhancement of facial shape, such as widening the upper midface (as in fat grafting but avoiding its issues) and inferior midface soft tissue contouring, creating narrowing the lower midface as desired; this allows powerful control of facial shape in the rejuvenation procedure.

The massive soft tissue mobilization and vertical repositioning create the need for careful surgical planning, extended incisions, and advanced closure techniques more complex than the original description. Overall, this technique maximizes the surgeon's abilities in primary rhytidectomy procedures and is preferred in revision procedures (-Fig. 1A, B).

It is for these reasons we present our advancements and nuances in creating the vertical platysma advancement technique as a variable, couture, advance of the original deep-plane rhytidectomy procedure.

\section{Conflict of Interest}

None.

\section{References}

1 Gordon NA, Toman J. Illusion of volume loss. Facial Plast Surg 2015;31(01):80-87

2 Tower J, Seifert K, Paskhover B. Longitudinal analysis of superficial midfacial fat volumes over a 10 -year period. Aesthetic Plast Surg 2018;42(04):995-1001
3 Tower JI, Seifert K, Paskhover B. Patterns of superficial midfacial fat volume distribution differ by age and body mass index. Aesthetic Plast Surg 2019;43(01):83-90

4 Skoog T. New Methods and Refinements in Plastic Surgery. Philadelphia, PA: Saunders; 1974

5 Mitz V, Peyronie M. The superficial musculo-aponeurotic system (SMAS) in the parotid and cheek area. Plast Reconstr Surg 1976;58 (01):80-88

6 Hamra ST. Composite rhytidectomy. Plast Reconstr Surg 1992;90 (01):1-13

7 Hamra ST. The deep-plane rhytidectomy. Plast Reconstr Surg 1990;86(01):53-61, discussion 62-63

8 Rohrich RJ, Pessa JE. The fat compartments of the face: anatomy and clinical implications for cosmetic surgery. Plast Reconstr Surg 2007;119(07):2219-2227, discussion 2228-2231

9 Litner JA, Adamson PA. Limited vs extended face-lift techniques: objective analysis of intraoperative results. Arch Facial Plast Surg 2006;8(03):186-190

10 Rees TD, Liverett DM, Guy CL. The effect of cigarette smoking on skin-flap survival in the face lift patient. Plast Reconstr Surg 1984; 73(06):911-915

11 Gordon NA, Rosenberg R. Deep plane rhytidectomy: technical modifications, nuances, and observations, a 17 year experience [Master's seminars]. Presented at the American Academy of Facial Plastic and Reconstructive Surgery Annual Meeting, September 5, 2012, Washington, DC

12 Barton FE Jr. Rhytidectomy and the nasolabial fold. Plast Reconstr Surg 1992;90(04):601-607

13 Gordon NA. Revision rhytidectomy: technique, modifications, and indications. Presented at the 10th International Symposium on Facial Plastic Surgery \& American Academy of Facial Plastic and Reconstructive Surgery, April 29, 2010, Hollywood, FL

14 Owsley JQ, Fiala TG. Update: lifting the malar fat pad for correction of prominent nasolabial folds. Plast Reconstr Surg 1997;100 (03):715-722

15 Yousif NJ, Gosain A, Matloub HS, Sanger JR, Madiedo G, Larson DL. The nasolabial fold: an anatomic and histologic reappraisal. Plast Reconstr Surg 1994;93(01):60-69

16 Paul MD, Calvert JW, Evans GR. The evolution of the midface lift in aesthetic plastic surgery. Plast Reconstr Surg 2006;117(06): 1809-1827

17 Matarasso A. Pseudoherniation of the buccal fat pad: a new clinical syndrome. Plast Reconstr Surg 1997;100(03):723-730, discussion 731-736

18 Zhang H-M, Yan Y-P, Qi K-M, Wang J-Q, Liu Z-F. Anatomical structure of the buccal fat pad and its clinical adaptations. Plast Reconstr Surg 2002;109(07):2509-2518, discussion 2519-2520

19 Mendelson BC, Tutino R. Submandibular gland reduction in aesthetic surgery of the neck: review of 112 consecutive cases. Plast Reconstr Surg 2015;136(03):463-471

20 Whetzel TP, Mathes SJ. The arterial supply of the face lift flap. Plast Reconstr Surg 1997;100(02):480-486, discussion 487-488

21 Jacono AA, Alemi AS, Russell JL. A meta-analysis of complication rates among different SMAS facelift techniques. Aesthet Surg J 2019;39(09):927-942

22 Parikh SS, Jacono AA. Deep-plane face-lift as an alternative in the smoking patient. Arch Facial Plast Surg 2011;13(04):283-285

23 Kamer FM, Song AU. Hematoma formation in deep plane rhytidectomy. Arch Facial Plast Surg 2000;2(04):240-242 